\title{
Direct Simulation and Experimental Study of Zigzag Maneuver of KCS in Shallow Water
}

\author{
Pablo M. Carrica*, Alireza Mofidi \\ IIHR-Hydroscience and Engineering \\ The University of Iowa, Iowa City, USA \\ Katrien Eloot, Guillaume Delefortrie \\ Flanders Hydraulics Research, Antwerp, Belgium
}

\begin{abstract}
The KCS container ship on a zigzag maneuver in shallow water is studied experimentally and numerically. The approach conditions are $F r=0.095$ and $H / T=1.2$. Experiments were performed in the shallow water towing tank at Flanders Hydraulics Research for the workshop on verification and validation of ship manoeuvring simulation methods SIMMAN 2014. CFD simulations were initially performed blind at the nominal rudder rate of $16.8 \mathrm{deg} / \mathrm{s}$ using direct discretization of moving rudder and propeller, including the tank bottom but neglecting walls. Grid studies were conducted for self-propulsion and the zigzag maneuver at the nominal rudder rate using grids of up to 71.3 million points. A simulation was then executed on the medium grid at the actual rudder experimental rate achieved $(8.35 \mathrm{deg} / \mathrm{s})$, and results analyzed. The grid study suggests that forces, moments and motions can be well predicted with coarser grids, and that medium grid results are very close to the fine grid, but flow details do not converge for the levels of refinement used. The verification results in large grid uncertainties, even though time evolutions of different variables show good grid convergence. CFD predictions match satisfactorily the experimental results for most variables, but under-predict yaw and yaw rate.
\end{abstract}

Keywords: Shallow water, Ship maneuvers, Experimental fluid dynamics, Computational fluid dynamics.

*Corresponding author. Pablo M. Carrica, 100 C. Maxwell Stanley Hydraulics Laboratory, The University of Iowa, IA 52241, USA. Email: pablo-carrica@uiowa.edu. 


\section{Introduction}

Commercial ship design has mostly been focused towards resistance and propulsion optimization to reduce operational costs. The maneuvering behavior of ships has not been treated with comparable attention, although resistance and propulsion optimization do not necessarily lead to better maneuvering capabilities. The idea that maneuvering performance should not be neglected lead to the IMO criteria MSC.137(76) (2002) for deep water. The maneuvering behavior of a ship becomes even more important when approaching a harbor. There the available water depth is limited, and the ship experiences forces due to the interaction with the environment (other ships, banks, etc.). Larger ship sizes lead to more restricted maneuvering space as the harbors and access channels cannot follow the pace of increasing ship sizes.

The problem is important in different regions around the world, including the Flemish region in Belgium, which are specifically concerned with the study of the ship maneuvering behavior in shallow and confined water. The main harbors in Belgium are all located in shallow and confined waters and the newest generation of Ultra Large Container Vessels call the ports of Zeebrugge and Antwerp. The admittance policy is based on real-time ship simulation studies which make use of the results of the shallow water maneuvering towing tank at Flanders Hydraulics Research (FHR), the research laboratory of the Flemish region in Belgium. This towing tank is a scientific collaboration with the Maritime Technology division of Ghent University. Shallow water benchmark data produced by FHR and Ghent University as a resource to validate experiments and CFD computations can be found in the report of the $27^{\text {th }}$ ITTC Manoeuvring Committee (2014). The benchmark data are currently being used by the international community to validate their own experiments or CFD computations. 
Use of CFD for maneuvering prediction is becoming more popular as numerical algorithms improve and computers gain in power. Due to the importance of viscous effects on maneuvering, Unsteady Reynolds Averaged Navier-Stokes (URANS) and Detached Eddy Simulation (DES) approaches are used rather than inviscid solvers. URANS/DES simulations replace the static and dynamic PMM experiments to obtain hydrodynamic derivatives and provide detail local flow physics around the hull under maneuvering motions (Sakamoto et al. 2012).

Among several possible approaches to perform CFD of maneuvering ships, direct CFD simulation is the most complex and the one least reliant on models, though it requires capabilities for self-propulsion, rotating propellers, moving rudders and full 6DOF in a free surface environment. Due to complexity and cost, there have been a limited number of direct CFD simulations for ship maneuvers. The first direct CFD simulations of ship maneuvering involved approximations to lighten the computational load. Several RANS maneuvering approaches for ships with modeled propellers have been attempted with various levels of success (Jacquin et al. 2006, Muscari et al 2008, Carrica et al. 2013). These approaches are cheaper than direct simulation of the propeller primarily because the time step is not limited by the stringent requirements to resolve the propeller flow. There are some quality experiments for validation of CFD of ship maneuvers. In SIMMAN 2008 experimental data for a surface combatant (DTMB 5415), very large crude carriers (KVLCC1 and KVLCC2) and KCS were reported for free model tests (Stern et al. 2011). In SIMMAN 2014 prediction capabilities of different ship maneuvering simulation methods, including systems-based and CFD, were tested blindly against experimental data for KVLCC2, KCS and DTMB 5415. In addition to the deep water IMO maneuvers, SIMMAN 
2014 also focused on maneuvering in shallow water. Most ships are designed for operation in deep water, but have to operate also in shallow water when entering harbor. A good discussion of the shallow water effects on resistance, forces and moments, form factor, and local flow field is described in Toxopeus (2013), who performed CFD calculations for the tanker KVLCC2. The results showed that resistance increases with water depth, but effects are most dramatic when operating at a drift angle, in which case lateral forces and moments increase considerably in shallow water. Shallow water results demonstrated that the flow separation is increased at the stern. Also, shallow water effects on flow separation are more significant in maneuvering conditions compared to straight ahead conditions. Through comparison with experiments, Toxopeus demonstrates that CFD can properly predict the trends observed when operating in shallow water.

Cura-Hochbaum and Uharek (2014) performed standard rudder maneuvers simulation for the KCS by using a mathematical model of Abkowitz type. They determined the hydrodynamic coefficients by means of virtual model tests performed with the RANS code Neptun. The results of the turning circle and zigzag tests in deep water fulfill all IMO recommendations with margin. For the shallow water case the results show larger advance and transfer values and much smaller overshoot and drift angles. Mikkelsen et al. (2014) performed simulations of pure sway PMM maneuvers using a RANS solver for KCS container ship with rudder and propeller. The propeller is modeled as a virtual disk adding momentum to the flow and forces to the ship. They also performed a mesh study which showed that monotonic and oscillatory convergence was achieved for the results.

Full direct CFD simulations of maneuvers of ships and submarines have been presented recently. 10/10 and 15/1 zigzag maneuvers of the Potsdam Model Basin (SVA) 
KCS model with moving rudder and rotating propeller were computed by Mofidi and Carrica (2014a). The simulations are performed at model scale in deep water, for approach velocity corresponding to $F r=0.26$, showing comparisons against towing tank zigzag tests. Shen et al. (2014) performed RANS simulations of zigzag and turn maneuvers of DTMB 5415M, KVLCC2 and KCS with moving rudder and propeller using overset grids in OpenFOAM. The approach showed great flexibility and the ability to perform complex computations with relatively coarse grids. Chase et al. (2013) and Martin et al. (2015) performed simulations of horizontal and vertical overshoots of the submarine models DARPA Suboff and ONR Body-1, respectively. In the case of ONR Body-1, extensive comparison with experimental data was performed, showing very good agreement.

Due to complexity and cost, verification studies for simulations of free running ships with directly moving propellers and rudders are not available in the literature. In this paper a study of a 20/5 modified zigzag maneuver for the KCS containership was performed in shallow water with depth to draft ratio $\frac{h}{T}=1.2$, including experimental fluid dynamics (EFD) and CFD using the fully implicit dynamic overset solver REX. Since there are some obstacles in performing standard zigzag maneuvers at small rudder angles for less stable or unstable ships like large container ships, modified maneuvers are executed to examine course keeping and maneuvering qualities. In case of fully loaded container ships standard zigzag maneuvers usually yields large overshoots that require very large basins to execute. Therefore, a modified zigzag maneuver is used to represent course keeping qualities in conditions similar to actual operations. 
Grid studies of the self-propulsion condition and the 20/5 modified zigzag maneuver are performed. In these computations the fully appended geometry is directly discretized accurately following the geometry in the experiments. This direct approach is expensive, but eliminates modeling of propellers or appendages. Direct simulation attempts to resolve all physics involved in the maneuver and the only aspect needing mathematical modeling is turbulence. The grid study is performed for the nominal rudder rate condition as part of a blind test. The rudder rate was then corrected to match the experimental value, and computations are repeated on the medium grid. Extensive comparisons are performed against EFD data for pitch, roll, yaw, yaw rate and rudder angles, and forces and moments in the propeller. The CFD work in this paper is an extension of the work by Mofidi and Carrica (2014b) on verification of a zigzag maneuver of KCS in shallow water.

\section{$\underline{\text { KCS geometry and conditions }}$}

KCS is a container ship designed by KRISO in Korea. Views of ship model and geometry are shown in Fig. 1, and main particulars of the model are listed in Table 1.

At the nominal self-propulsion approach speed of $0.62 \mathrm{~m} / \mathrm{s}$ in model scale the Reynolds number is $\operatorname{Re}=\frac{U_{0} L_{p p}}{v}=2.74 \times 10^{6}$, corresponding to $\mathrm{Fr}=\frac{U_{0}}{\sqrt{g L_{p p}}}=0.095$, where $U_{0}$ is the ship service speed and $L_{p p}$ is the length between perpendiculars. $\rho$ is the water density, $v$ is the kinematic viscosity of water and $g$ is the acceleration of gravity. The reference coordinate system attached to the ship follows the standard in the software used (REX), with $x$ the longitudinal axis pointing to stern, $y$ pointing to starboard and $z$ pointing upwards with $z=0$ at 
calm water plane. The Earth system is coincident with the ship system at rest at the initial condition, and is denoted by $\left(X_{e}, Y_{e}, Z_{e}\right)$. For EFD and to show results, however, the standard naval architecture system of coordinates is used, where $x$ points to the bow along the ship axis, $y$ points to starboard and $z$ points downward. The yaw is then defined positive to starboard, the rudder angle is positive when turning to port, pitch is positive bow up, and roll positive to starboard. Units are in model scale unless otherwise stated.

The metacentric height is $11.39 \mathrm{~mm}$, corresponding to a vertical location of the center of gravity of $63.5 \mathrm{~mm}$ above the waterline in model scale. The propeller, designed by SVA, has 5 blades, variable pitch and a diameter of $0.15 \mathrm{~m}$. The propeller's principal particulars are given in Table 2, showing that for the case in this paper the pitch to diameter ratio is 0.997 . The rudder is a semi-balanced horn design with details provided also in Table 2 .

Experiments and simulations are performed for a fairly shallow water condition with depth to draft ratio $h / T=1.2$. The modified $20 / 5$ zigzag is carried out at constant propeller rotational speed, obtained from self-propulsion at the approach speed, and executing the rudder initially to starboard at the nominal rudder rate of $16.8 \mathrm{deg} / \mathrm{s}$. These were the conditions for the blind simulations requested in SIMMAN 2014, but experiments were finally performed with a rudder rate of $8.35 \mathrm{deg} / \mathrm{s}$ and slightly different moments of inertia, see Table 1.

\section{$\underline{\text { Experimental methods }}$}

The free running tests discussed in this article were carried out in the Towing Tank for Maneuvers in Shallow Water at FHR. This tank has a usable length of $68 \mathrm{~m}$, a width of $7 \mathrm{~m}$ and a water depth that can be varied to a maximum of $0.5 \mathrm{~m}$. The towing tank can be operated in an unmanned way, enabling 24/7 test execution. The towing tank carriage can be used both in 
captive mode (the motions of the ship are forced in the three horizontal degrees of freedom), or in free running mode. In the latter, the towing carriage is used to accelerate the ship model and to track its position once released, see Fig. 2. After test completion, the ship model is caught again and decelerated. In between tests, the ship model is always moved to the next position in a captive way, after which a waiting period of $2000 \mathrm{~s}$ is needed for the water in the tank to settle. The limited available width restricts the possible maneuvers that can be performed in the tank.

In the frame of the SIMMAN 2014 workshop (Simman 2014) both captive and free running tests were executed with the KCS model, contributed by SVA. The free running tests, see Table 3, are all executed with depth to draft ratio $h / T=1.2$. Due to geometric limitations, only the initial part of the turning circles could be carried out. Also alternative zigzag tests were executed to limit the lateral path of the ship model and to limit possible tank wall collision and effects, though wall effects were not quantified. During a test, the position of the carriage in the horizontal plane is monitored throughout the experiment. The position of the carriage is used to track position of the ship model. The relative position of the ship model with respect to the carriage is determined by laser reflections between the carriage and the ship model, with the carriage following the ship and acting as reference position. The control loop is designed such that linear and angular deviations between carriage and ship model are within $1 \mathrm{~mm}$ and 0.1 degrees, respectively. During post processing the test results are transformed to the ship frame of reference, so that the real behavior of the ship model is presented, including any possible heel or drift angles. Also, the sinkage at fore and aft locations are monitored, resulting in complete information to determine the 6 DOF position of the ship model with a sampling frequency of 40 Hz. For the SIMMAN 2014 tests, the propeller rate, thrust and shaft torque were also recorded. 
The tests were executed in 2010 following the relevant ITTC procedures. However, after the SIMMAN 2014 workshop the tests results were reprocessed because the interval of the running average was too large to make comparisons with the CFD results, in particular the harsh filtering of the rudder angle made it impossible to detect the rudder executions during the zigzag test. Moreover, due to friction issues, the nominal rudder rate of $16.8 \mathrm{deg} / \mathrm{s}$ was not achievable during the experimental test.

Ten test repetitions were performed to evaluate uncertainty. ITTC recently issued a new guideline (ITTC Quality Systems Manual, 2014) on sensitivity analysis for free running tests. The new guideline discusses propagation of the uncertainty on the initial conditions, which at FHR fluctuate little due to the captive acceleration procedure used as described previously in this section. Even if the initial conditions are not well controlled (within certain limits), the KVLCC2 example presented in the ITTC guideline shows that the number of test repetitions is far more important in determining the uncertainty than possible deviations in initial conditions. It is interesting to note, in addition, that experiments at FHR show much larger standard deviations for tests on KVLCC2 than for KCS experiments, even though the acceleration and release mechanisms used in the experiments is the same for both models and thus initial conditions are similar for both models. A more detailed discussion on this topic is provided by Eloot et al. (2015).

Figure 3 shows the measured yaw angles, rudder angles and computed yawing rates for ten tests of the 20/5 zigzag maneuver with an initial rudder execution to starboard. As previously stated, the initial conditions are very well controlled with a very repeatable experimental rudder angle rate, resulting in also highly repeatable experimental results. Once the prescribed yaw angle change of 5 degrees is detected for the first rudder execute, small deviations in rudder 
angle setting and yaw angle change can be observed. The time difference in zero crossing between the ten tests is largest at the second rudder angle execute, but remains smaller than 1 second (5\% of the maneuver time to the zero crossing). The shown yaw rate is computed differentiating the measured yaw rate. This procedure amplifies noise present in the actual yaw rate, which produces oscillations in the yaw rate. The statistical results of the ten tests are shown in Table 4 with relative deviations situated between $0.46 \%$ for the period and $2.75 \%$ for the first overshoot. For comparisons with CFD simulations, Run 10 of the $20 / 5$ zigzag test starting to starboard was used.

\section{Mathematical and numerical modeling}

The computations are performed with the ship hydrodynamics CFD code REX, a merge of the codes CFDShip-Iowa v4.5 (Carrica et al. 2007a, 2007b, 2010) and the more modern code Magnus, both developed at The University of Iowa. REX solves the unsteady RANS free surface equations using a single-phase level set approach, with capabilities for DES and DDES. The turbulence is modeled by a blended $k-\omega / k-\varepsilon$ with shear stress transport (SST) turbulence model.

The domain is discretized using curvilinear multiblock structured grids that can overlap. The overset capabilities are fully dynamic, which allows the code to simulate large-amplitude motions and control surface deflections. The overset connectivity is obtained at run time using Suggar (Noack 2005).

A fourth-order upwind biased discretization is used for the convective terms, while a secondorder central is used for the viscous terms. The second-order backward Euler scheme is used for discretization of temporal terms. Incompressibility is enforced with a projection method that 
provides strong pressure/velocity coupling. 6DOF capabilities are incorporated with a hierarchy of bodies. Grids belonging to the ship (parent body) move according to the 6DOF equations, while the propeller (child 1) and rudder (child 2) follow the ship but can have motion relative to it. The fluid flow equations are solved in an earth-fixed inertial reference system while the rigidbody equations are solved in the ship system, and forces and moments are projected appropriately to perform the integration of the rigid-body equations of motion, which are solved iteratively. The incompressible Navier-Stokes equations are non-dimensionalized using the reference velocity and length, $U$ and $L$, usually the ship's speed and length between perpendiculars, and corresponding fluid properties. Though not used in this work, REX has additional functionalities including capabilities to compute bubbly flows including air entrainment models, to simulate stratified flows, and to handle deployment, tow and recovery of auxiliary craft through cable models involving the multibody dynamics code MagnusDyn.

Feedback controllers can be used for speed, heading, course and roll control while open loop controllers are used for maneuvers. In this paper an open loop controller is used to move the rudder during the zigzag maneuver, and a proportional-integral (PI) speed controller is used to act on the propeller rotational speed to achieve the target speed for self-propulsion computations

$n=P e+I \int_{0}^{t} e d t$

where $n$ is the rotational speed of the propeller (in RPS), $P$ and $I$ are the proportional and integral gains, respectively, and $e$ is the error respect to the speed target value $e=U_{\text {target }}-U_{\text {ship }}$

Details of the modeling and numerical methodologies can be found in Mofidi and Carrica (2014), Martin et al. (2015), and references therein. 


\section{$\underline{\text { Grid and simulation design }}$}

Figure 4 shows the overset grid topology at the solid surfaces and cross-sections detailing the free surface and propeller/rudder refinements. The overset grid system consists of 38 grid blocks for medium and fine grids, including grids for hull, propeller, rudder, refinements and background. These 38 blocks are distributed in 168 and 352 processors for medium and fine grids, respectively, as listed in Table 5 for the fine grid. The coarse grid consists of 40 grid blocks distributed in 71 processors, needing two more blocks to ensure enough overlap in the area of the hull stern shaft hub and the region above the water in air. The addition of these additional blocks is not expected to have a significant impact on the results, since grid spacings on the added blocks are similar to those of the neighboring grids. This still deviates from the systematic grid refinement paradigm, but so does in general the overset grid technique by requiring sufficient overlap. The total number of grid points for the coarse, medium and fine grids are 8.7, 24.6 and 71.3 million, respectively. About two-thirds of the blocks are used for refinement to resolve the flow around the propeller and rudder and on wake region to resolve vortical structures and flow features in those regions, and better capture the hull/propeller/rudder interactions critical to predict maneuvering forces and moments. Since propeller blades and rudder are very close to each other and also the gap between the rudder's horn and moving part of the rudder is very thin, overlapping in those regions requires grids fine enough to obtain valid interpolations between the grids.

Appendages are handled using overset grids. The grids have a collar surface grid that conforms to the hull, and other surface grids that conform to the appendage itself. From these surface grids volume grids are generated using hyperbolic solvers provided by the commercial grid generation software Gridgen. Grid spacing on solid boundaries is designed to handle 
boundary layers up to $R e=20 \times 10^{6}$ with the SST turbulence model integrating all the way to the wall. Once all grid blocks are generated Suggar is used to generate valid overset domain connectivity information, initially as a preprocessing step to assure proper overlap and grid quality, and then dynamically at run time as the ship, propeller and rudder move.

Table 5 also shows the hierarchy of bodies used to run the simulation. The ship body is solved as a rigid body in 6DOF, and contains all grids defining the geometry and the refinements that follow the ship in all degrees of freedom. Rudder and propeller grids are children to the ship, and move according to the control laws for these appendages. All propeller grids rotate together as a rigid body with one degree of freedom, the rotational angle controlled by the speed controller. These propeller grids form a dynamic overset group. The rudder is constructed similarly and forms a second dynamic overset group, with rudder angle controlled by the zigzag controller. A third dynamic overset group is formed by all ship and refinement grids belonging to the ship body excluding the rudder and propeller. Finally, the grids connecting the ship to the farfield boundary conditions, called Background and Refinement grids in Table 5, follow the ship but are not allowed to pitch, heave or roll so that the refinements in these grids designed to resolve the free surface and bottom stay horizontal.

For the medium grid the simulations were performed using 3 Suggar groups, as described in Carrica et al. (2010). Though Suggar uses only 4 parallel threads, 8 cores were assigned to each group to guarantee enough memory is available for Suggar to run. In the machine used to perform these computations there are $27 \mathrm{~Gb}$ of accessible memory per 16-core node, then leaving $13.5 \mathrm{~Gb}$ of memory available for each Suggar group to run. The first 168 cores (10.5 nodes) were assigned to CFD processes, one per MPI rank, while the next 24 cores (1.5 nodes) were used to run Suggar, for a total of 192 cores required for the simulations. Similar 
considerations were employed for the fine grid, which used 352 CFD cores and 48 Suggar cores, and for the coarse grid, which used 71 cores for CFD and 9 for Suggar.

The preprocessing step then requires generating the grids, setting up inputs and running Suggar with appropriate boundary conditions, testing that valid interpolations exist (no orphan points) for different propeller and rudder angles, and finally running Usurp (Boger and Dryer 2006) to obtain the information of overset panels for force integration.

Before the simulations are started, a pre-processing hydrostatic computation is performed to obtain the longitudinal location of the center of gravity, the mass and the static wetted area of the ship in static condition with zero resulting pitching moment and the weight balanced by buoyancy. Though these values are usually provided for design conditions, CFD values can deviate a small amount (typically less than $0.1 \%$ ) due to the fact that the geometry was discretized and may be slightly off due to CAD approximations. The resulting nominal, CFD and EFD values for the longitudinal location of the center of gravity and the displacement are shown in Table 6.

The simulation does not include the walls of the towing tank where the experiments were performed, but uses a no-slip boundary condition at the bottom. All simulations are performed in the earth coordinate system, thus the boundary conditions are inlet at the upstream and side boundaries $\left(U=V=W=0, \frac{\partial P}{\partial n}=0\right)$, fixed non-slip at the bottom (in this case same as inlet), moving non-slip at all solid surfaces $\left(\boldsymbol{U}=\dot{\boldsymbol{x}}, \frac{\partial P}{\partial n}=0\right)$ and exit at the downstream boundary $\left(\frac{\partial^{2} U}{\partial n^{2}}=0, \frac{\partial^{2} V}{\partial n^{2}}=0, \frac{\partial^{2} W}{\partial n^{2}}=0, \frac{\partial P}{\partial n}=0\right)$.

The self-propulsion computations are performed using a PI controller on the propeller rotational speed to achieve the target velocity $U_{0}=0.62 \mathrm{~m} / \mathrm{s}$ as described by Eqs. (1) and (2). 
The self-propulsion condition is attained when the ship speed and propeller rotational speed reach steady state. This requires an acceleration phase to overcome the ship inertia, as well as development of the boundary layer and wave field. To improve convergence to self-propulsion, the inertia of the ship was decreased by a factor of 10 during the computations, which increases the acceleration of the ship but has no effect on the results once the steady-state is reached. For zigzag simulations the actual inertia of the system is used.

The zigzag maneuvers are restarted from the self-propulsion condition, letting the ship free in 6DOF and executing the rudder according to a rudder controller that limits the rudder rate to a specified value and changes rudder direction according to the check heading imposed in the maneuver. During the zigzag maneuvers the propeller rotational speed is maintained constant at the self-propulsion value. CFD simulations were initially performed blind at the nominal rudder rate of $16.8 \mathrm{deg} / \mathrm{s}$. A simulation was then executed on the medium grid at the actual rudder rate achieved in the experiments, and comparisons and analysis of the results were performed.

A total of 52000 time steps with increment $\Delta t=1.56 \mathrm{~ms}$ were used to get approximately one period for the 20/5 modified zigzag maneuver for coarse and medium grids and a total of 53000 time steps with $\Delta t=1.3 \mathrm{~ms}$ were used for fine grid. Runs were performed on an SGI ICE-X computer. Times to complete $27 \mathrm{~s}$ of full scale time were approximately 250 hours of wall clock time for the fine grid, and 170 hours for the medium grid and coarse grids,.

\section{Grid studies for self-propulsion and zigzag maneuver at the nominal rudder rate}

A grid study was performed to estimate the grid numerical uncertainties of the solutions for self-propulsion and for the $20 / 5$ zigzag maneuver at the nominal rudder rate according to the procedure presented in Stern et al. (2001) and Wilson et al. (2004). This simulation was performed in a blind fashion, and then compared with experimental data at SIMMAN 2014. Due 
to experimental limitations already described, the rudder rate in the experiments was lower than the nominal rate. The simulations were repeated at the experimental rudder rate using the medium grid, but the grid study was not repeated due to cost and the considerable similarity for both conditions. Both CFD and EFD results for the experimental rudder rate are presented in the next section.

The first step for estimation of the grid uncertainty $\left(U_{G}\right)$ is the convergence study. Solutions on three systematically refined grids with refinement ration $R_{G}$ are required:

$$
r_{G}=\frac{\Delta x_{2}}{\Delta x_{1}}=\frac{\Delta x_{3}}{\Delta x_{2}}
$$

where the subscripts 1,2 and 3 represent the fine, medium and coarse grids and $\Delta x$ is the grid spacing. Three convergence conditions are possible, defined as

$$
R_{G}=\frac{\varepsilon_{12}}{\varepsilon_{23}}=\frac{S_{2}-S_{1}}{S_{3}-S_{2}}=\left\{\begin{array}{cl}
0<R_{G}<1 & : \text { Monotonic convergence } \\
R_{G}<0 & : \text { Oscillatory convergence } \\
R_{G}>1 & : \text { Divergence }
\end{array}\right.
$$

In grid divergence condition no uncertainty can be estimated. In oscillatory grid convergence the uncertainty is estimated by

$$
U_{G}=\left|1 / 2\left(S_{U}-S_{L}\right)\right|
$$

where $S_{U}$ and $S_{L}$ are the oscillation maximums and oscillation minimums of the solutions from the grids. In monotonic convergence the generalized Richardson extrapolation is used to estimate the grid error $\delta_{R E_{G}}^{*}$ and the order of accuracy $P_{G}$,

$$
\begin{gathered}
\delta_{R E_{G}}^{*}=\frac{\varepsilon_{G_{21}}}{r_{G}^{P_{G}}-1} \\
P_{G}=\frac{\ln \left(\varepsilon_{G_{32}} / \varepsilon_{G_{21}}\right)}{\ln \left(r_{G}\right)}
\end{gathered}
$$


When $\delta_{R E_{G}}^{*}$ and $P_{G}$ are known the grid uncertainty is estimated. There are two procedures to estimate the grid uncertainty based on the range of the correction factor, $C_{G}$, which is given as:

$$
C_{G}=\frac{r_{G}^{P_{G}}-1}{r_{G}^{P_{G e s t}}-1}
$$

where $P_{\text {Gest }}$ is the limiting or theoretical accuracy of the applied numerical method. If $C_{G} \approx 1$ the solutions are close to the asymptotic range. In this case the sign of the error is known, and the numerical error $\delta_{S N}^{*}$, benchmark $S_{C}$ and uncertainty $U_{G C}$ can be calculated as

$$
\begin{aligned}
& \delta_{S N}^{*}=C_{G} \delta_{R E_{G}}^{*} \\
& S_{C}=S-\delta_{S N}^{*} \\
& U_{G C}=\left\{\begin{array}{c}
\left\{\left(2.4\left(1-C_{G}\right)^{2}+0.1\right)\left|\delta_{R E_{G}}^{*}\right|, \quad\left|1-C_{G}\right|<0.125\right. \\
\left|1-C_{G}\right|\left|\delta_{R E_{G}}^{*}\right|,\left|1-C_{G}\right| \geq 0.125
\end{array}\right.
\end{aligned}
$$

If $C_{G}>>1$ only the numerical uncertainty is calculated as

$$
U_{G}=\left\{\begin{array}{l}
\left(9.6\left(1-G_{C}\right)^{2}+1.1\left|\delta_{R E_{G}}^{*}\right|,\left|1-G_{C}\right|<0.125\right. \\
\left(2\left|1-G_{C}\right|+1\right)\left|\delta_{R E_{G}}^{*}\right|,\left|1-G_{C}\right| \geq 0.125
\end{array}\right.
$$

Table 7 summarizes the three grids and the design $y^{+}$of the first boundary layer grid point. An appropriate refinement ratio of $r_{G}=\sqrt{2}$ is used here.

Grid studies were performed on propeller forces and moments and motion parameters. It should be noted that the solutions on all three grids are run only to the point where the zigzag maneuver covers the first two overshoots.

Figure 5 shows the evolution of the propeller rotational speed and the ship velocity as selfpropulsion is attained for all three grids. Self-propulsion at the target speed of $0.62 \mathrm{~m} / \mathrm{s}$ is 
achieved at 338.7 RPM, which compares to the experimental value of 331.5 RPM. Forces and torque on the propeller are shown in Fig. 6. Table 8 shows verification results in propeller RPM, forces, torque and pitch and roll angles at self-propulsion.

The grid study shown in Table 8 shows monotonic convergence for RPM with $R_{G}=0.33$, propeller torque and side force with $R_{G}$ of 0.15 and 0.4 respectively. The pitch angle also converged monotonically with $R_{G}$ of 0.54 . The roll angle and propeller thrust show oscillatory convergence with $R_{G}=-0.23$ and -0.48 , respectively. The $U_{G}$ is around $1 \% S_{2}$ for all variables, except for roll and pitch angles and the propeller side force, which are one order of magnitude larger closer to $10 \% S_{2}$, though these variables are very small in self-propulsion and thus subject to large relative errors. The average of the grid uncertainty for the most relevant self-propulsion variables (RPM, propeller thrust and torque) is about $U_{G}=0.75 \%$, suggesting that the effects of the grid changes are small on the results for the present range of grid sizes.

Validation was performed for propeller RPM, thrust and torque, and pitch and roll angles at self-propulsion. The comparison error, validation uncertainty, experimental data uncertainty, and simulation numerical uncertainty are shown in Table 9. The comparison error $E$ is the difference between experimental data $D$ and simulation $S$ values

$$
E=D-S=\delta_{D}-\left(\delta_{S M}+\delta_{S N}\right)
$$

where $\delta_{S M}$ and $\delta_{S N}$ are modelling and numerical errors respectively. To characterize if validation has been achieved, the comparison error $E$ is compared to the validation uncertainty $U_{V}$ given by:

$$
U_{V}^{2}=U_{D}^{2}+U_{S N}^{2}
$$

where $U_{D}$ is experimental uncertainty and $U_{S N}$ is simulation numerical uncertainty. If $|E|<U_{V}$, the combination of all the errors in $\mathrm{D}$ and $\mathrm{S}$ is smaller than $U_{V}$ and validation is achieved at the 
$U_{V}$ level. If $|E| \geq U_{V}$, modelling improvements are needed (Wilson et al. 2004). Validation is the process of estimating the simulation modeling uncertainty $U_{S M}$ by using experimental data and estimating the magnitude and sign of the modelling error $\delta_{S M}$. Therefore, the experimental errors and uncertainties must be considered in addition to the numerical errors and uncertainties in calculation of the total validation uncertainty.

An experimental uncertainty analysis was conducted for the validation parameters including key elemental errors for propeller RPM, propeller torque and thrust, and pitch and roll angles at self-propulsion. Table 10 summarizes the experimental errors and total uncertainties of the variables listed above. The total uncertainty is computed following the methodology described in ANSI/ASME PTC 19.1 (2005) as:

$$
\begin{gathered}
U_{B_{\text {Tot }}}=\sqrt{B_{L}^{2}+B_{R}^{2}+B_{S}^{2}} \\
U_{P_{\text {Tot }}}=\sqrt{P_{\text {Curve fit }}^{2}+P_{\text {Repeatability }}^{2}} \\
U_{D}=t t_{v, 95} \sqrt{U_{B_{T o t}}^{2}+U_{P_{T o t}}^{2}}
\end{gathered}
$$

where $U_{B_{T o t}}$ and $U_{P_{T o t}}$ represent total bias or systematic error and total precision or random error, respectively. Calibration errors comprise those elemental errors occurred during the calibration of measuring system, and include the standard or reference value used in calibration $\left(B_{S}\right)$, and the calibration process or curve fit $\left(P_{\text {Curve fit }}\right) . B_{L}$ and $B_{R}$ in equation (15) represent linearity and resolution errors, respectively. The repeatability error $\left(P_{\text {Repeatability }}\right)$ is calculated based on the ten experiment runs for each variable. Details of the experimental uncertainties are presented in Table 10. 
As can be seen in Table 9, for $R P M,|E|>U_{V}$ such that it is not validated at $|E|=2 \% D$. For $Q_{P}, T_{p}, \theta$ and $\varnothing,|E|<U_{V}$ such that these variables are validated at uncertainty validation levels of $U_{V}=3.26 \% D, 3.01 \% D, 935 \% D$ and $63.8 \% D$, respectively. The pitch angle is validated at a very large relative validation uncertainty level due to the very small absolute value and large $U_{S N}=61 \% D$. The same occurs, but to a lesser extent, with the roll angle.

Table 11 summarizes the results of the grid study for the 20/5 zigzag maneuver at the nominal rudder rate. Verification parameters are maximums and minimums of roll, pitch, yaw and drift angles, roll rate, and yaw rate, and propeller thrust, torque and side force. The grid study shows monotonic convergence for maximum roll angle, maximum and minimum pitch angles, minimum yaw angle and propeller side force with $R_{G}$ of $0.013,0.42,0.13,0.35$ and 0.074, respectively. The minimum roll angle, minimum and maximum roll rate show oscillatory convergence with $R_{G}=-0.38, R_{G}=-0.014$ and $R_{G}=-0.054$, respectively. Maximum yaw angle and maximum and minimum yaw rate also display oscillatory convergence with $R_{G}=$ $-0.43, R_{G}=-0.3$ and $R_{G}=-0.69$, respectively. Maximum and minimum drift angle variables also show oscillatory convergence with $R_{G}=-0.44$ and $R_{G}=-1.74$. The propeller thrust at maximum and minimum yaw rate converged oscillatorily with $R_{G}=-5.6$ and $R_{G}=-0.33$. Propeller torque at minimum yaw rate also shows oscillatory convergence with $R_{G}=-0.018$.

Several propeller forces and torque parameters show monotonic divergence for the zigzag maneuver, though they converged monotonically for the self-propulsion grid study. In addition the minimum drift angle exhibits oscillatory divergence. The nature of the time evolution dependence of the flow, and thus forces and integrated motion quantities, complicates the grid study, highlighting the difficulties of using the classic grid convergence procedures designed for steady-state problems when analyzing transient flows like a zigzag maneuver. Inspection of Figs. 
7 to 11 show good grid convergence, with remarkable similarity between medium and fine grids, but with the coarse grid deviating more, most notably for the roll angle shown in Fig. 9. This may indicate that the coarse grid is too coarse for asymptotic behavior, and that an even finer grid is needed for the study, which with the refinement ratio $r_{G}=\sqrt{2}$ would result in approximately $200 \mathrm{M}$ grid points, prohibitive for the computational resources available to the authors.

The grid uncertainties are higher than for the self-propulsion analysis, with $U_{G}$ for the parameters that exhibit monotonic convergence ranging from $-17.26 \% S_{2}$ to $2.69 \% S_{2}$ in which the minimum is for maximum pitch angle and the maximum is for propeller torque at maximum yaw rate. The average grid uncertainty for monotonic convergence is $U_{G}=2.6 \%$, slightly higher than the experimental standard deviation. Grid uncertainties are higher for parameters showing oscillatory convergence. Notice that validation cannot be performed for the zigzag maneuver since the available experimental data are for a different rudder rate.

Figures 7 and 8 show time histories of yaw, yaw rate and rudder angle for the 20/5 zigzag maneuver. All grids predict very similar values of yaw and yaw rate as the peak values for these variables converged in monotonic or oscillatory form. Figure 9 shows the roll angles for all grids. As can be seen, the difference between the peaks in roll angle for coarse and medium grids is about $16.6 \%$ and between the coarse and fine grids this value is about $16.8 \%$. It is clear that the medium and fine grids are predicting the roll angles for the duration of the maneuver with less than $0.25 \%$ difference. Figure 10 shows the evolution of the pitch angle and roll rate during the maneuver. Notice that pitch angles are very small and the differences between grids are most noticeable for this quantity, but the trends are still consistent for coarse, medium and fine grids. The roll rate shows trends more consistent with the roll angle, where the medium and fine grid 
results are almost coincident throughout the maneuver, but the coarse grid sees differences with the other two grids that increase in time. The ship absolute velocity and drift angle are shown in Fig. 11. After one zigzag period the ship has not yet reached periodic behavior, with the absolute velocity still decreasing and the drift angle increasing. Here the drift angle is very similar between the grids, but the velocity drops faster for the fine grid than for the medium and coarse grids.

Time histories of propeller thrust and torque for the zigzag maneuver are shown in Fig. 12. Predicted thrust and torque are higher for the coarse grid respect to the medium and fine grids. The propeller torque is the quantity that seems most dependent on the grid refinement used, while the lateral forces are very similar for all grids. Rudder resistance and yaw moment are shown in Fig. 13. Every time the rudder is executed there is a minimum in rudder resistance as the rudder aligns with the flow as it turns, predicted consistently by all grids.

Figure 14 shows velocity and pressure contours at self-propulsion, maximum yaw rate and minimum yaw rate for all grids. These contours are at downstream of the propeller at $x / L=$ 1.01. The results show that finer grids produce more detailed flow field, resolving smaller scales, and thus do not converge in finer features as the grid is resolved. The velocity field is especially revealing since it doesn't produce the fast transients that the pressure sees as it adjusts to conserve mass. This result is expected since LES regions will only converge as the grid approaches the dissipation length, requiring grids orders of magnitude finer than those used in this study.

\section{$\underline{\text { Results and discussion at the experimental rudder rate }}$}


The experimental rudder rate of $8.35 \mathrm{deg} / \mathrm{s}$ is used to compare experimental and CFD results for the 20/5 zigzag maneuver. Recall that the nominal rudder rate, used in the grid study, was $16.8 \mathrm{deg} / \mathrm{s}$ which was used in Figs. 7-11, already discussed in the context of the grid study.

The time histories of yaw and rudder angles, depicted in Fig. 7, show that use of the experimental rudder angle improves dramatically the phase response of the simulation, which matches very well the experiments. The maximum and minimum yaw amplitudes increase respect to the faster nominal rudder rate results, but still are lower than the experimental values. The maximum yaw rate, shown in Fig. 8, remains underpredicted by CFD and does not improve when using the slower experimental rudder execution rate respect to the nominal rudder rate. In general, the agreement between CFD predictions and EFD measurements is very good in terms of time to check yaw, period and reach, with some underestimation of the yaw rate and thus the yaw overshoot. The yaw angles are 12.3 and 12.7 degrees for the EFD first and second overshoot, respectively, while CFD results show 10.7 and 10.3 degrees, respectively, implying an underestimation in the CFD predictions of $13 \%$ and $18.9 \%$, respectively. The period of the maneuver is $39.9 \mathrm{~s}$, with CFD predicting a value only $0.2 \%$ smaller at $39.8 \mathrm{~s}$. The maximum yaw rate peaks reaches to $1.99 \mathrm{deg} / \mathrm{s}$ for EFD and $1.62 \mathrm{deg} / \mathrm{s}$ for CFD. These values as well as other important parameters of the maneuver are shown in Table 11.

The yaw and yaw rate reach quickly an apparently periodic condition after the $2^{\text {nd }}$ rudder execute around $8 \mathrm{~s}$ after starting the maneuver, though results of ship velocity discussed later show that the periodic condition has not been reached. Note that the experimental data has a starting yaw rate of 0.04 degrees/s, which causes some level of drift of the data towards starboard. Conversely, the CFD yaw rate is exactly zero at the beginning of the simulation since 
the self-propulsion computation used to obtain the approach speed was restricted from any yaw motion.

Satisfactory reasons for the underprediction by CFD of the yaw rate and consequent underprediction of yaw angles have not been found, and more research is needed to understand the reasons for these discrepancies, mostly in view of the good agreement between CFD and EFD for all other parameters. Possible sources of error include under-prediction of rudder yaw moment due to separation in CFD possibly not present in the experiments, over-prediction of the hull yaw moments, effects of neglecting the walls of the tank, the differences in the inertia properties between CFD and EFD, etc. The first two sources of errors, and probably others potential errors not yet identified, can be mostly attributed to turbulence modeling. The effects of the walls are purely geometric and can be evaluated through appropriate inclusion of the walls in the simulation, though is not done in the present work.

Roll rate and roll, pitch, absolute velocity and drift angles, shown in Figs. 9-11, show good agreement between $\mathrm{CFD}$ and EFD, though the minimum roll angle is under predicted. As expected, the use of the experimental rudder rate dramatically improves the CFD predictions compared to EFD. Notice that the pitch angles are very small but the CFD trends are still consistent with experiments, which show a bow up tendency every time the rudder is executed. The average trim is about 0.005 degrees lower for CFD than EFD. The CFD pitch fluctuations, though small, are larger in amplitude for CFD than EFD, possibly due to wave reflection on the boundaries. The roll rate, related to the roll damping, is also very consistent with experimental data. The drift angle also agrees very well between EFD and CFD, both in amplitude and phase. As expected, the drift angle is in phase with the yaw rate. 
The velocity of the ship decreases rapidly as the maneuver progresses. Even though there is some uncertainty on how accurately the nominal approach speed has been achieved in EFD, CFD follows the behavior of EFD fairly well. Every time the rudder is executed the ship deceleration decreases, evidence that when rudders are actuated the resistance decreases, providing some level of excess thrust from the propeller. For the duration of the maneuver the zigzag has not reached periodic behavior, since the velocities are still dropping.

Though increased resistance due to drift and roll are contributors to the speed loss in a maneuvering ship, changes in propeller forces have an influence tending to compensate by increasing thrust as the velocity decreases and the local propeller advance coefficient decreases. The process is, however, much more complicated than a simple change in advance coefficient since the wake velocity changes considerably through the maneuver.

Time histories of propeller thrust and torque for both zigzag maneuvers are shown in Fig. 15, where excellent agreement between CFD and EFD is evident. The average thrust increases with time slowly as the ship speed and thus the advance coefficient decrease. However, large lowfrequency fluctuations occur, with increases in thrust and torque that correlate negatively with yaw rate. While this indicates that thrust and torque are maximum when the ship turns to port at maximum rate, it is also evidence of the asymmetries that a single screw propeller can cause, in this case with peak to peak fluctuations of $10 \%$ of the average thrust and torque. In addition, high frequency fluctuations in thrust and torque that correlate to the blade passage frequency are presented, clear both in CFD. The EFD data acquisition rate is not high enough to resolve this blade passage frequency, presenting considerable aliasing, but the amplitudes appear to be reasonable close to the CFD predictions. Recall the propeller rotational speed is approximately $331.5 \mathrm{RPM}$, which results in a blade passage frequency of $27.5 \mathrm{~Hz}$, too high to be resolved by 
the data acquisition rate of 40 samples/s. Thrust and torque fluctuations due to the passage of the blades through different regions of the wake are described in detail in Castro et al. (2011).

An animation of the zigzag maneuver in real time in model scale is shown in Electronic Annex I, where the vortical structures are visualized as isosurfaces of the second invariant of the velocity gradient tensor $Q$. Vortical structures and free surface are colored with axial velocity. The bottom of the tank is not shown in the video, but the interaction between the propeller/rudder flow with the bottom surface is clear at this low $h / T=1.2$ condition. Since the ship is operating at a low velocity and therefore the Froude number is small, the free surface is not disturbed much by the ship motion except in the wake.

Figures 16 and 17 show vorticity magnitude at instantaneous horizontal sections at the depth of the propeller hub center $(z / R=0)$ and $0.7 R$ above and below it, and axial cross sections at the trailing edge of the rudder. The solutions are taken at points where the yaw is minimum (approximately $31 \mathrm{~s}$ in Fig. 7) and maximum (49 s through the maneuver), where the yaw rate is minimum (25 s in Fig. 8) and maximum (42 s), and when the yaw is zero turning to port (22 s in Fig. 7) and turning to starboard (40 s). An animation of a stroboscopic visualization of the propeller/rudder interaction, similar to the procedure followed in a cavitation tunnel with propellers, is shown in Electronic Annex II. This animation was produced with results from the fine grid at the nominal rudder rate, but the fundamental interaction is the same as with the medium grid at the experimental rudder rate. The interaction between propeller and rudder is considerable, with the propeller trailing edge, tip and hub vortices hitting the rudder and being stretched by it. Notice that Figures 16 and 17, and Electronic Annexes I and II are shown in the CFD frame of reference. 
The bottom portion of the rudder $(z / R=-0.7)$ operates mostly in attached condition, but showing massive stall at the minimum yaw rate point. Notice for the maximum yaw rate and the zero yaw turning to starboard points that the interaction between the trailing edge vortices and the rudder causes distinct stretching and strengthening of the vorticity going from leading edge to trailing edge of the rudder. The top section of the rudder $(z / R=0.7)$ is more complex since the rudder horn is fixed and adds additional interactions. The presence of the rudder horn makes the system to work as a cambered foil with a smaller angle of attack, but still showing signs of separation for the points of maximum yaw, minimum yaw rate and zero yaw to port. The cross section at $(z / R=0)$ shows considerably stronger vorticity, caused by the presence of the hub vortex. This hub vortex/propeller interaction does not necessarily cause separation, as seems to occur at the points of minimum yaw, minimum yaw rate and zero yaw turning to port. Separation is observed, however, at maximum yaw, while the bottom portion of the rudder separates at minimum yaw. This difference is caused by the swirl introduced by the propeller, which flows starboard to port at the bottom of the propeller and port to starboard at the top, adding to the drift occurring during the maneuver. An interesting phenomenon occurs at the maximum and minimum yaw rate at $z / R=0$, corresponding to approximately $24 \mathrm{~s}$ into the animation in Electronic Annex II for minimum yaw rate. In Electronic Annex II the tip vortices interact with the strong bilge vortex generated by the interaction of bottom and the ship as it turns. The bilge vortex, clearly observable in Fig. 17 at maximum yaw rate in the axial cross section but also noticeable at minimum yaw rate, has axial vorticity co-rotating with the tip vortices and maximum strength at maximum turning rate. This generates considerable axial vorticity and instability in the vortical structures, as can be seen in Electronic Annex II starting at $20 \mathrm{~s}$. 
The boundary layer at different instants is depicted in Fig. 18, as cross sections colored with vorticity magnitude. The interaction with the bottom and the drift velocity cause the generation of strong bilge vortices that interact with the propeller, most notably at the minimum and maximum yaw rate conditions happening from the zero yaw points until the minimum and maximum yaw rates are reached. The relative side velocity between the bow and the bottom generates a strong vortex there.

Figures 16, 17 and 18 are evidence of the complexity of the turbulent flow in the propeller/rudder region during a maneuver. As the flow reaches the rudder, it already has high levels of turbulence at different scales produced initially by the ship boundary layer and interaction with the bottom, and augmented by the propeller action. The adequacy of RANS and hybrid RANS/LES methods to predict correct rudder forces and moments under these types of flows needs to be investigated, as proper rudder forces are paramount to obtain good predictions for ship maneuvers.

\section{Conclusions and future work}

A 20/5 zigzag maneuver for the container ship KCS was studied experimentally and numerically. Ten experimental runs were completed to estimate precision errors, each with a semi-captive self-propulsion stage and a subsequent free zigzag maneuver. The CFD analysis includes grid studies for the self-propulsion stage and the zigzag maneuver at the nominal rudder rate, and a simulation with the medium grid for the actual achieved experimental rudder rate.

This study shows that experimental repeatability is excellent, with standard deviations below $3 \%$ for all key variables throughout the maneuver. This high repeatability is achieved by 
controlling very well the initial conditions. The resulting data are of high quality, suitable for CFD validation.

This work presents the first grid study for direct CFD simulations of self-propulsion and free maneuvers. The self-propulsion grid study shows good grid convergence for the most relevant variables (RPM and propeller thrust and torque), though higher uncertainty for the propeller side force. The grid study for the zigzag maneuver was performed using the same procedures designed for steady-state processes, and results in much higher grid uncertainties than the selfpropulsion study, with several parameters exhibiting oscillatory convergence or even divergence, highlighting the difficulties of performing grid studies complex on naturally transient ship maneuvers.

Comparison of CFD results with EFD data are satisfactory for the self-propulsion condition, with errors below $2.7 \%$ for all relevant variables. For the zigzag maneuver at the experimental rudder rate CFD compares very well with the experiments for all variables, except yaw and yaw rate, which exhibit errors of about $15 \%$ for yaw on the overshoots and around $20 \%$ for the yaw rate, in both cases lower than EFD data. Possible reasons for these underpredictions include underprediction of rudder yaw moment and overprediction of the hull yaw moment, likely due to improper separation caused by errors in the turbulence model. In addition, the walls of the tank were neglected in the CFD simulations, which could have a significant role on the discrepancies observed with EFD because of the higher blockage occurring in shallow water conditions. Analysis of the effects of the walls in shallow water zigzag maneuvers is left for future research.

While studying maneuvers with EFD is the closest approach to mimic reality, it time consuming to prepare an instrumented model with controllable rudder and propeller, setup the facilities to perform the experiments, and run the tests for the period of the experimental 
campaign. CFD has proven capable of good results and provides significant insight of the

hydrodynamics occurring during the maneuver that could be very difficult to measure, like velocity and pressure fields, three dimensional vortical structures, etc.

\section{$\underline{\text { Acknowledgments }}$}

This work was partially supported by the Office of Naval Research under grants N0001414-1-0134 and N00014-14-1-0302, with Drs. Thomas Fu and Ki-Han Kim as program managers.

\section{$\underline{\text { References }}$}

$27^{\text {th }}$ ITTC Manoeuvring Committee, 2014. "Final Report and Recommendations to the $27^{\text {th }}$ ITTC Conference", $27^{\text {th }}$ ITTC Conference, Copenhagen, Denmark.

ANSI/ASME Power Test Codes-PTC 19.1 (2005). Test Uncertainty, American Society of Mechanical Engineers, New York, New York, USA.

Boger D, Dryer J, 2006. "Prediction of Hydrodynamic Forces and Moments for Underwater Vehicles Using Overset Grids," 2006-1148, 44 ${ }^{\text {th }}$ AIAA Aerospace Sciences Meeting and Exhibit, Reno, Nevada, USA.

Carrica PM, Wilson RV, Stern F, 2007a. "An Unsteady Single-Phase Level Set Method for Viscous Free Surface Flows," Int. J. Num. Meth. Fluids 53, 229-256.

Carrica PM, Wilson RV, Noack R, Stern F, 2007b. "Ship Motions using Single-Phase Level Set with Dynamic Overset Grids," Comput. Fluids 36, 1415-1433.

Carrica PM, Huang J, Noack R, Kaushik D, Smith B, Stern F, (2010a) "Large-Scale DES Computations of the Forward Speed Diffraction and Pitch and Heave Problems for a Surface Combatant," Comput. Fluids 39, 1095-1111.

Carrica PM, Ismail F, Hyman M, Bhushan S, Stern F, 2013. "Turn and Zigzag Maneuvers of a Surface Combatant Using a URANS Approach with Dynamic Overset Grids," J. Marine Sci. Technol. 18, 166-181.

Castro AM, Carrica PM, Stern F, 2011. Full scale self-propulsion computations using discretized propeller for the KRISO container ship KCS," Comput. Fluids 51, 35-47.

Chase N, Michael T, Carrica PM, 2013. "Overset simulations of a Submarine in Towed, SelfPropelled and Maneuvering Conditions," Int. Shipbuilding Prog. 60, 171-205.

Cura-Hochbaum A, Uharek S, 2014. "Prediction of the Maneuvering Behavior of the KCS Based on Virtual Captive Test" SIMMAN 2014 Workshop on Verification and Validation of Ship Maneuvering Simulation Methods, Copenhagen, Denmark.

Eloot K, Delefortrie G, Vantorre M, Quadvlieg F, 2015. "Validation of Ship Manoeuvring in Shallow Water Through Free Running Tests.” OMAE 2015, St. John's, Canada. 
ITTC Quality Manual, 2014. "ITTC - Recommended Procedures and Guidelines: Uncertainty Analysis for Free Running Model tests (7.5-02-06-05)". Available at http://ittc.info/downloads/Quality\%20systems\%20manual/Index/index.pdf

International Maritime Organization. 2002. "Standards for Ship Maneuverability," Resolution MSC.137(76).

Jacquin E, Guillerm PE, Drouet A, Perdon P, Alessandrini B, 2006. "Simulation of Unsteady Ship Maneuvering Using Free-Surface RANS Solver," $26^{\text {th }}$ ONR Symp. Naval Hydrodyn., Rome, Italy.

Martin JE, Michael T, Carrica PM, 2015. "Submarine Maneuvers Using Direct Overset Simulation of Appendages and Propeller and Coupled CFD/Potential Flow Propeller Solver," J. Ship Res. (in press).

Mikkelsen H, Sanchez F, Knudsen S, 2014. "RANS Simulation of the KCS Container Ship in Pure Sway Motions," Proc. SIMMAN 2014 Workshop on Verification and Validation of Ship Maneuvering Simulation Methods, Copenhagen, Denmark.

Mofidi A, Carrica PM, 2014a. "Simulations of Zigzag Maneuvers for a Container Ship with Direct Moving Rudder and Propeller," Comput. Fluids 96, 191-203.

Mofidi A, Carrica PM, 2014b. "Verification of Zigzag Maneuver for a Container Ship with Direct Moving Rudder and Propeller," SIMMAN 2014 Workshop on Verification and Validation of Ship Maneuvering Simulation Methods, Copenhagen, Denmark.

Muscari R, Broglia R, Di Mascio A, 2008. "Trajectory prediction of a self-propelled hull by unsteady RANS computations," $27^{\text {th }}$ ONR Symp. Naval Hydrodyn., Seoul, Korea.

Noack R, 2005. "SUGGAR: a general capability for moving body overset grid assembly," AIAA paper 2005-5117, $17^{\text {th }}$ AIAA CFD Conf., Toronto, ON.

Sakamoto N, Carrica PM, Stern F, 2012. "URANS simulations of static and dynamic maneuvering for surface combatant: part 1 . Verification and validation for forces, moment, and hydrodynamic derivatives," J. Mar. Sci. Technol. 17, 422-445.

Shen Z, Wan D, Carrica PM, 2014. "RANS Simulations of Free Maneuvers with Moving Rudders and Propellers using Overset Grids in OpenFOAM," Proc. SIMMAN 2014 Workshop on Verification and Validation of Ship Maneuvering Simulation Methods, Copenhagen, Denmark.

Stern F, Wilson RV, Coleman HV, Paterson EG, 2001. "Comprehensive approach to verification and validation of CFD simulations-Part 1\&2: methodology and procedures," ASME J. Fluids Eng. 124, 793-802.

Stern F, Agdrup K, Kim SY, Hochbaum AC, Rhee KP, Quadvlieg F, Perdon P, Hino T, Broglia R, Gorski J, 2011. "Experience from SIMMAN 2008 - The First Workshop on Verification and Validation of Ship Maneuvering Simulation Methods," J. Ship Res. 55, 135-147.

Simman 2014, Workshop on Verification and Validation of Ship Manoeuvring Simulation Methods, Lyngby, Denmark, December 8-10, simman2014.dk.

Toxopeus, S., L. 2013. "Viscous-Flow Calculations for KVLCC2 in Deep and Shallow Water," Comput. Meth. Appl. Sci. 29, 151-169. 
Wilson R, Shao J, Stern F, 2004. "Discussion: Criticism of the "Correction Factor" Verification Method,” ASME J Fluids Eng. 126, 704-706. 


\begin{tabular}{|c|c|c|}
\hline & Nomenclature & \\
\hline $\begin{array}{l}\mathrm{Fr}=\text { Froude number } \\
\operatorname{Re}=\text { Reynolds number } \\
U_{0}=\text { ship service speed } \\
\rho=\text { water density } \\
v=\text { kinematic viscosity of water } \\
\mathrm{h}=\text { depth of water } \\
\mathrm{T}=\text { draft of ship } \\
k=\text { turbulence kinetic energy } \\
\varepsilon=\text { TKE dissipation rate } \\
\omega=\text { specific dissipation rate } \\
n=\text { propeller rotational speed }\end{array}$ & $\begin{array}{l}C_{G}=\text { correction factor } \\
P_{G e s t}=\text { theoretical accuracy } \\
\delta_{S N}^{*}=\text { numerical error } \\
S_{C}=\text { numerical benchmark } \\
U_{G C}=\text { uncertainty } \\
y^{+}=\text {wall distance } \\
E=\text { comparison error } \\
D=\text { experimental data value } \\
S=\text { simulation data value } \\
\delta_{D}=\text { experimental error } \\
\delta_{S M}=\text { modeling error }\end{array}$ & $\begin{array}{l}Q=\text { second invariant of the velocity } \\
\text { gradient tensor } \\
\phi=\text { roll angle } \\
\dot{\phi}=\text { roll rate } \\
\psi=\text { yaw angle } \\
\dot{\psi}=\text { yaw rate } \\
\beta=\text { drift angle } \\
T_{P_{x}}=\text { propeller thrust } \\
F_{P_{y}}=\text { propeller side force } \\
Q_{P}=\text { propeller torque } \\
\theta=\text { pitch angle }\end{array}$ \\
\hline $\begin{array}{l}\mathrm{P}=\text { proportional gain } \\
\mathrm{I}=\text { integral gain }\end{array}$ & $\begin{array}{l}\delta_{S N}=\text { numerical error } \\
U_{V}=\text { validation uncertainty }\end{array}$ & $\left(X_{e}, Y_{e}, Z_{e}\right)=$ earth system \\
\hline $\mathrm{e}=$ velocity error to target value & $U_{D}=$ experimental uncertainty & Delta $=|\max |-|\min |($ EFD $)$ \\
\hline$U_{G}=$ grid uncertainty & $\begin{array}{l}U_{S N}=\text { simulation numerical } \\
\text { uncertainty }\end{array}$ & \\
\hline$R_{G}=$ refinement ratio & $t_{v}=$ student's t variable & \\
\hline$\Delta_{x}=$ grid spacing & $\delta_{P}=$ precision error & \\
\hline $\begin{array}{l}S_{U}=\text { oscillation maximum of } \\
\text { solution } \\
S_{L}=\text { oscillation minimum of } \\
\text { solution } \\
\delta_{R E_{G}}^{*}=\text { generalized Richardson } \\
\text { extrapolation grid error } \\
P_{G}=\text { order of accuracy }\end{array}$ & $\begin{array}{l}\delta_{B}=\text { bias error } \\
\delta_{L}=\text { linearity error } \\
\delta_{C a}=\text { calibration error } \\
\delta_{S t}=\text { standard error } \\
g=\text { acceleration of gravity }\end{array}$ & \\
\hline
\end{tabular}


Table 1: Main particulars of the KCS model.

\begin{tabular}{lccc}
\hline Main Particulars & & Model Scale (CFD/EFD) & Full Scale \\
\hline Length of waterline & $L_{w l}(m)$ & 4.414 & 232.5 \\
Length between & $L_{p p}(m)$ & 4.367 & 230 \\
perpendiculars & $B_{w l}(m)$ & 0.611 & 32.2 \\
Maximum beam of & $T(m)$ & & \\
waterline & $h(m)$ & 0.205 & 10.8 \\
Draft & $\Delta\left(m^{3}\right)$ & 0.246 & 19 \\
Depth & $\Delta /\left(L_{p p} B_{w l} T\right)$ & 0.3562 & 52022 \\
Displacement & $S_{w}\left(m^{2}\right)$ & 0.651 & 0.651 \\
Block coefficient $(\mathrm{CB})$ & & 3.4357 & 54.5 \\
Wetted area $\boldsymbol{w} / \boldsymbol{o}$ rudder & & 0.984 & 0.985 \\
Midship section coefficient & & \\
(CM) & & $-1.48 /-1.49$ & -1.48 \\
Longitudinal center of & & & \\
Buoyancy & $G M(m)$ & 0.011 & 0.6 \\
Metacentric height & $K_{x x} / B$ & $0.40 / 0.45$ & 0.4 \\
Moment of inertia & $\left.K_{z z} / L_{p p}\right), F w d+$ & $0.25 / 0.24$ & 0.25 \\
Moment of inertia & $U(\mathrm{~m} / \mathrm{s})$ & 0.62 & 4.5 \\
Service speed & $F r$ & 0.095 & 0.095 \\
Froude number & $S C$ & 52.667 & 1 \\
Scale & &
\end{tabular}


Table 2: Details of propeller and horn rudder.

\begin{tabular}{lll}
\hline Model & SVA & Full Scale \\
\hline Scale & 52.667 & 1 \\
Propeller & & \\
Type & Controllable Pitch & Fixed Pitch \\
No. of Blades & 5 & 5 \\
$\mathrm{D}(\mathrm{m})$ & 0.150 & 7.9 \\
$\mathrm{P} / \mathrm{D}(0.7 \mathrm{R})$ & 1.000 & 0.997 \\
Rotation & Right Hand & Right Hand \\
Hub Ratio & 0.227 & 0.180 \\
$A_{e} / A_{0}$ & 0.700 & 0.800 \\
Rudder & & \\
S rudder $\left(m^{2}\right)$ & 0.0415 & 115 \\
Lat. Area $\left(m^{2}\right)$ & 0.0196 & 54.45 \\
Nominal turning rate $(\mathrm{deg} / \mathrm{s})$ & 16.8 & 2.32 \\
\hline
\end{tabular}


Table 3: KCS free running tests performed at FHR in the frame of SIMMAN2014.

\begin{tabular}{|l|c|c|c|c|}
\hline Test & $\begin{array}{c}\text { Nominal } \\
\text { approach } \\
\text { speed }(\mathrm{m} / \mathrm{s})\end{array}$ & $\begin{array}{c}\text { Nominal } \\
\text { propeller rate } \\
\text { (rpm) }\end{array}$ & Repetitions & $\begin{array}{c}\text { Sampling } \\
\text { frequency } \\
\text { (Hz) }\end{array}$ \\
\hline Start of 35 degree turn to port & 0.62 & 331.5 & 7 & 40 \\
\hline Start of 35 degree turn to starboard & 0.62 & 331.5 & 6 & 40 \\
\hline 10/2.5 zigzag test, starting to port & 0.62 & 331.5 & 11 & 40 \\
\hline 10/2.5 zigzag test, starting to starboard & 0.62 & 331.5 & 10 & 40 \\
\hline 20/5 zigzag test, starting to port & 0.62 & 331.5 & 10 & 40 \\
\hline $\mathbf{2 0 / 5}$ zigzag test, starting to starboard & $\mathbf{0 . 6 2}$ & $\mathbf{3 3 1 . 5}$ & $\mathbf{1 0}$ & $\mathbf{4 0}$ \\
\hline
\end{tabular}


Table 4: Averages and standard deviations of key quantities for ten repeated 20/5 zigzag tests with start to starboard.

\begin{tabular}{|l|c|r|r|r|r|}
\hline \multicolumn{1}{|c|}{ Quantity } & Unit & Mean & $\begin{array}{c}\text { Standard } \\
\text { Deviation }\end{array}$ & Relative & \multicolumn{1}{c|}{ Delta } \\
\hline Period & \multicolumn{1}{c|}{$(\mathrm{s})$} & 40 & 0.183 & $0.46 \%$ & 0.60 \\
\hline Time to 1st heading deviation (HD) & \multicolumn{1}{c|}{$(\mathrm{s})$} & 7 & 0.125 & $1.78 \%$ & 0.40 \\
\hline Travelled distance to 1st HD & $(\mathrm{m})$ & 4.316 & 0.090 & $2.09 \%$ & 0.29 \\
\hline Time between 1st HD and max heading $(\mathrm{MH})$ & $(\mathrm{s})$ & 6.17 & 0.157 & $2.54 \%$ & 0.60 \\
\hline Travelled distance between 1st HD and MH & $(\mathrm{m})$ & 3.692 & 0.082 & $2.23 \%$ & 0.31 \\
\hline Time between 2nd HD and max heading (MH) & $(\mathrm{s})$ & 6.11 & 0.110 & $1.80 \%$ & 0.30 \\
\hline Travelled distance between 2nd HD and MH & $(\mathrm{m})$ & 3.421 & 0.062 & $1.80 \%$ & 0.15 \\
\hline Max. heading 1 & $\left({ }^{\circ}\right)$ & 11.990 & 0.330 & $2.75 \%$ & 1.02 \\
\hline Max. heading 2 & $\left.{ }^{\circ}\right)$ & -12.739 & 0.234 & $1.84 \%$ & 0.81 \\
\hline
\end{tabular}


Table 5: Details of the fine grid system and decomposition information.

\begin{tabular}{lllll}
\hline Grid & Size & Grid Points & Belongs to body & Processors \\
\hline Boundary layer starboard 1 & $340 \times 77 \times 125$ & $3273 \mathrm{~K}$ & Ship & 18 \\
Boundary layer starboard 2 & $340 \times 81 \times 74$ & $2038 \mathrm{~K}$ & Ship & 10 \\
Stern starboard & $85 \times 67 \times 71$ & $404 \mathrm{~K}$ & Ship & 2 \\
Hub starboard & $99 \times 57 \times 71$ & $401 \mathrm{~K}$ & Ship & 2 \\
Boundary layer port1 & $340 \times 77 \times 125$ & $3273 \mathrm{~K}$ & Ship & 18 \\
Boundary layer port2 & $340 \times 81 \times 74$ & $2038 \mathrm{~K}$ & Ship & 10 \\
Stern port & $85 \times 67 \times 71$ & $404 \mathrm{~K}$ & Ship & 2 \\
Hub port & $99 \times 57 \times 71$ & $401 \mathrm{~K}$ & Ship/Propeller & 2 \\
Propeller hub & $142 \times 44 \times 128$ & $800 \mathrm{~K}$ & Ship/Propeller & 4 \\
Propeller blades & $5 \times 111 \times 71 \times 104$ & $5 \times 820 \mathrm{~K}$ & Ship/Propeller & 20 \\
Propeller tips & $5 \times 71 \times 51 \times 99$ & $5 \times 401 \mathrm{~K}$ & Ship & 10 \\
Refinement wake & $111 \times 191 \times 190$ & $4028 \mathrm{~K}$ & Ship & 20 \\
Refinement & $340 \times 200 \times 142$ & $9656 \mathrm{~K}$ & Ship (surge, sway, yaw only) & 48 \\
Background & $255 \times 198 \times 128$ & $6463 \mathrm{~K}$ & Ship (surge, sway, yaw only) & 36 \\
Rudder stem refinement & $85 \times 63 \times 153$ & $819 \mathrm{~K}$ & Ship & 4 \\
Rudder stem starboard & $85 \times 77 \times 125$ & $818 \mathrm{~K}$ & Ship & 4 \\
Rudder stem port & $85 \times 77 \times 125$ & $818 \mathrm{~K}$ & Ship & 4 \\
Rudder stem top & $77 \times 63 \times 85$ & $412 \mathrm{~K}$ & Ship & 2 \\
Rudder stem bottom & $167 \times 57 \times 85$ & $809 \mathrm{~K}$ & Ship & 4 \\
Refinement gap top & $36 \times 145 \times 77$ & $402 \mathrm{~K}$ & Ship & 2 \\
Rudder gap & $102 \times 49 \times 247$ & $1235 \mathrm{~K}$ & Ship/Rudder & 7 \\
Rudder top & $159 \times 50 \times 51$ & $405 \mathrm{~K}$ & Ship/Rudder & 2 \\
Rudder starboard & $297 \times 66 \times 125$ & $2450 \mathrm{~K}$ & Ship/Rudder & 14 \\
Rudder port & $297 \times 66 \times 125$ & $2450 \mathrm{~K}$ & Ship/Rudder & 14 \\
Rudder cap top & $99 \times 57 \times 71$ & $401 \mathrm{~K}$ & Ship/Rudder & 2 \\
Refinement propeller/rudder & $283 \times 242 \times 213$ & $14587 \mathrm{~K}$ & Ship & 75 \\
Refinement rudder bottom & $170 \times 165 \times 29$ & $813 \mathrm{~K}$ & Ship & 4 \\
Refinement rudder port & $165 \times 29 \times 170$ & $813 \mathrm{~K}$ & Ship & 4 \\
Refinement rudder starboard & $165 \times 29 \times 170$ & $813 \mathrm{~K}$ & Ship & 4 \\
Refinement rudder top & $165 \times 170 \times 29$ & $813 \mathrm{~K}$ & Ship & $\mathbf{3 5 2}$ \\
Total & & $\mathbf{7 1 . 3 ~ M}$ & & \\
& & & & \\
\hline
\end{tabular}


Table 6: Hydrostatic results for medium grid.

\begin{tabular}{llll}
\hline Parameter & CFD & EFD (nominal) & EFD (actual) \\
\hline Displacement $\left(\boldsymbol{m}^{\mathbf{3}}\right)$ & 0.3528 & 0.3562 & 0.3561 \\
Longitudinal Center of & 0.5098 & 0.5092 & 0.5096 \\
Gravity $(\mathrm{m})$ & & & \\
\hline
\end{tabular}


Table 7: Grid dimensions and $y^{+}$values for refinement study.

\begin{tabular}{ccc}
\hline Grid & Grid Points & $\boldsymbol{y}^{+}$ \\
\hline 1- Coarse & $8.7 \mathrm{M}$ & 1.4 \\
2- Medium & $24.6 \mathrm{M}$ & 1 \\
3- Fine & $71.3 \mathrm{M}$ & 0.7 \\
\hline
\end{tabular}


Table 8: Grid convergence for forces, moment and motions at self-propulsion. $\delta_{G}^{*}, U_{G C}$ and $U_{G}$ are $\% S_{2}$.

\begin{tabular}{|c|c|c|c|c|c|c|c|c|c|c|c|}
\hline Variable & $\begin{array}{c}\text { Coarse } \\
\text { Grid }\end{array}$ & $\begin{array}{c}\text { Medium } \\
\text { Grid }\end{array}$ & $\begin{array}{c}\text { Fine } \\
\text { Grid }\end{array}$ & $\boldsymbol{R}_{\boldsymbol{G}}$ & $\boldsymbol{P}_{\boldsymbol{G}}$ & $\boldsymbol{C}_{\boldsymbol{G}}$ & $\boldsymbol{\delta}_{\boldsymbol{G}}^{*}$ & $\boldsymbol{U}_{\boldsymbol{G}}$ & $\boldsymbol{S}_{\boldsymbol{C}}$ & $\boldsymbol{U}_{\boldsymbol{G}}$ & $\begin{array}{c}\text { Convergence } \\
\text { Type }\end{array}$ \\
\hline$R P M$ & 338.87 & 338.76 & 338.7 & 0.33 & 3.2 & 2.03 & 0.01 & 0.005 & 338.7 & 0.02 & Monotonic \\
\hline$Q_{P}$ & 132.1 & 126.8 & 125.4 & 0.26 & 3.8 & 2.8 & 1.1 & 0.71 & 125.4 & 1.15 & Monotonic \\
\hline$F_{P_{y}}$ & 0.0726 & 0.088 & 0.0925 & 0.29 & 3.5 & 2.4 & -5.1 & 2.99 & 0.0925 & 8.67 & Monotonic \\
\hline$\theta$ & -0.0111 & -0.0133 & -0.0145 & 0.54 & 1.75 & 0.83 & -9.02 & -1.80 & -0.0145 & -10.8 & Monotonic \\
\hline$T_{P}$ & 5.48 & 5.4 & 5.352 & 0.68 & 1.1 & 0.47 & 1.01 & 1.15 & 5.344 & 1.04 & Monotonic \\
\hline$\varnothing$ & 0.135 & 0.108 & 0.121 & -0.23 & & & & & & 12.5 & Oscillatory \\
\hline
\end{tabular}


Table 9: Validation of thrust, torque, motions and propeller RPM at self-propulsion. Percentages are respect to the EFD data.

\begin{tabular}{|c|c|c|c|c|c|c|c|}
\hline Variable & $\boldsymbol{S}$ & $\boldsymbol{E}$ & $\boldsymbol{D}$ & $\boldsymbol{E} \%$ & $\boldsymbol{U}_{\boldsymbol{V}} \%$ & $\boldsymbol{U}_{\boldsymbol{D}} \%$ & $\boldsymbol{U}_{\boldsymbol{S N}} \%$ \\
\hline$R P M$ & 338.7 & 331.5 & -7.18 & -2.2 & 0.743 & 0.742 & 0.0164 \\
\hline$Q_{P}$ & 125.4 & 128.9 & 3.53 & 2.7 & 3.26 & 2.72 & 1.786 \\
\hline$\theta$ & -0.0145 & -0.0024 & 0.012 & -514.1 & 934.9 & 932.9 & 60.98 \\
\hline$T_{P}$ & 5.352 & 5.348 & 0.004 & 0.08 & 3.01 & 2.06 & 2.192 \\
\hline$\varnothing$ & 0.121 & 0.307 & 0.186 & 60.6 & 63.8 & 63.6 & 4.401 \\
\hline
\end{tabular}


Table 10: Experimental uncertainties of thrust, torque, motions and propeller RPM at self-propulsion.

\begin{tabular}{|c|c|c|c|c|c|c|c|c|}
\hline Variable & $\boldsymbol{B}_{\boldsymbol{L}}$ & $\boldsymbol{B}_{\boldsymbol{R}}$ & $\boldsymbol{B}_{\boldsymbol{S}}$ & $\boldsymbol{P}_{\text {Curve fit }}$ & $\boldsymbol{P}_{\text {Repeatability }}$ & $\boldsymbol{U}_{\boldsymbol{B}}$ & $\boldsymbol{U}_{\boldsymbol{P}}$ & $\boldsymbol{U}_{\boldsymbol{D}}$ \\
\hline$R P M$ & 1 & 0.5 & - & - & 0.029 & 1.12 & 0.029 & 2.46 \\
\hline$Q_{P}$ & 0.97 & 0.332 & 0.3225 & 0.583 & 1.026 & 1.075 & 1.18 & 3.51 \\
\hline$\theta$ & - & 0.01 & - & - & 0.00043 & 0.01 & 0.00043 & 0.022 \\
\hline$T_{P}$ & 0.025 & 0.015 & - & - & 0.0405 & 0.029 & 0.0405 & 0.11 \\
\hline$\varnothing$ & - & 0.005 & - & - & 0.0886 & 0.005 & 0.0886 & 0.195 \\
\hline
\end{tabular}


Table 11: Grid convergence for forces, moment and motions variables for the zigzag maneuver at nominal rudder rate. $\delta_{G}^{*}, U_{G C}$ and $U_{G}$ are $\% S_{2}$.

\begin{tabular}{|c|c|c|c|c|c|c|c|c|c|c|c|}
\hline Variable & $\begin{array}{c}\text { Coarse } \\
\text { Grid } \\
\end{array}$ & $\begin{array}{c}\text { Medium } \\
\text { Grid }\end{array}$ & $\begin{array}{l}\text { Fine } \\
\text { Grid } \\
\end{array}$ & $R_{G}$ & $P_{G}$ & $C_{G}$ & $\delta_{G}^{*}$ & $U_{G C}$ & $s_{C}$ & $U_{G}$ & $\begin{array}{c}\text { Convergence } \\
\text { Type } \\
\end{array}$ \\
\hline$\emptyset_{\max }$ & 2.074 & 1.73 & 1.725 & 0.013 & 12.57 & 77.08 & 0.25 & 0.25 & 1.72 & 0.51 & Monotonic \\
\hline$\emptyset_{\min }$ & -1.78 & -1.87 & -1.84 & -0.38 & & & & & & -2.5 & Oscillatory \\
\hline$\theta_{\max }$ & -0.002 & -0.00295 & -0.00335 & 0.42 & 2.5 & 1.375 & -13.56 & -3.7 & -0.0034 & -17.26 & Monotonic \\
\hline$\theta_{\min }$ & -0.02174 & -0.02026 & -0.02006 & 0.13 & 5.77 & 6.4 & -0.002 & 0.001 & 8.44 & 0.003 & Monotonic \\
\hline$\psi_{\max }$ & 8.29 & 8.44 & 8.38 & -0.43 & & & & & & -0.9 & Oscillatory \\
\hline$\psi_{\min }$ & -8.23 & -8.51 & -8.60 & 0.35 & 3.03 & 1.86 & -0.004 & 0.002 & 1.67 & 0.02 & Monotonic \\
\hline$\dot{\phi}_{\max }$ & 1.36 & 1.53 & 1.52 & -0.054 & & & & & & 5.53 & Oscillatory \\
\hline$\dot{\phi}_{\min }$ & -1.43 & -1.492 & -1.490 & -0.0135 & & & & & & 0.66 & Oscillatory \\
\hline$\dot{\psi}_{\max }$ & 1.38 & 1.47 & 1.46 & -0.106 & & & & & & -14.15 & Oscillatory \\
\hline$\dot{\psi}_{\min }$ & -1.52 & -1.60 & -1.58 & -0.277 & & & & & & -8.26 & Oscillatory \\
\hline$\beta_{\max }$ & 1.579 & 1.673 & 1.632 & -0.44 & & & & & & 2.8 & Oscillatory \\
\hline$\beta_{\min }$ & -1.79 & -1.82 & -1.77 & -1.74 & & & & & & -1.17 & $\begin{array}{l}\text { Oscillatory } \\
\text { Divergence }\end{array}$ \\
\hline $\boldsymbol{T}_{\boldsymbol{P}_{X, \dot{\psi} \max }}$ & 5.31 & 5.21 & 5.08 & 1.411 & -0.99 & -0.29 & 2.58 & 11.42 & 5.08 & 14 & $\begin{array}{l}\text { Monotonic } \\
\text { Divergence } \\
\end{array}$ \\
\hline $\boldsymbol{T}_{\boldsymbol{P}_{X, \dot{\psi}_{\min }}}$ & 4.92 & 4.91 & 4.81 & 15.356 & -7.88 & -0.93 & 2.09 & 4.33 & 4.81 & 6.42 & $\begin{array}{l}\text { Monotonic } \\
\text { Divergence }\end{array}$ \\
\hline $\boldsymbol{F}_{\boldsymbol{P}_{y, \dot{\psi}} \max }$ & 0352 & 0.352 & 0.316 & 170.33 & -14.82 & -0.99 & 10.16 & 20.4 & 0.32 & 30.54 & $\begin{array}{l}\text { Monotonic } \\
\text { Divergence } \\
\end{array}$ \\
\hline $\boldsymbol{F}_{\boldsymbol{P}_{y, \dot{\psi}_{\text {min }}}}$ & -0.486 & -0.459 & -0.438 & 0.77 & 0.75 & 0.298 & 4.55 & -10.73 & -0.44 & -15.29 & Monotonic \\
\hline $\boldsymbol{Q}_{P, \dot{\psi}_{\text {max }}}$ & 131.45 & 125.39 & 122.3 & 0.518 & 1.90 & 0.93 & 2.5 & 0.19 & 122.25 & 2.69 & Monotonic \\
\hline $\boldsymbol{Q}_{P, \dot{\psi}_{\min }}$ & 120.88 & 119.75 & 116.52 & 2.84 & -3.01 & -0.65 & 2.69 & 6.85 & 116.52 & 9.54 & $\begin{array}{l}\text { Monotonic } \\
\text { Divergence }\end{array}$ \\
\hline
\end{tabular}


Table 12: Parameters for the $20 / 5$ modified zigzag maneuver.

\begin{tabular}{lllllll}
\hline & $\begin{array}{l}\text { First } \\
\text { Overshoot } \\
\text { (degrees) }\end{array}$ & $\begin{array}{l}\text { Second } \\
\text { Overshoot } \\
\text { (degrees) }\end{array}$ & $\begin{array}{l}\text { Yaw } \\
\text { Period }(\mathbf{s})\end{array}$ & $\begin{array}{l}\text { First Yaw Rate } \\
\text { Peak } \\
\text { (degrees/s) }\end{array}$ & $\begin{array}{l}\text { Second Yaw Rate } \\
\text { Peak } \\
\text { (degrees/s) }\end{array}$ & $\begin{array}{l}\text { Yaw Rate }\left(\dot{\psi}_{\max }-\dot{\boldsymbol{\psi}}_{\min }\right) / 2 \\
(\text { degrees/s) }\end{array}$ \\
\hline EFD & 12.3 & 12.7 & 39.9 & 1.95 & 1.98 & 1.99 \\
CFD & $10.7(-13 \%)$ & $10.3(-18.9 \%)$ & $39.8(-0.2 \%)$ & $1.44(-26.1 \%)$ & $1.62(-18.2 \%)$ & $1.63(-18.1 \%)$ \\
\hline
\end{tabular}




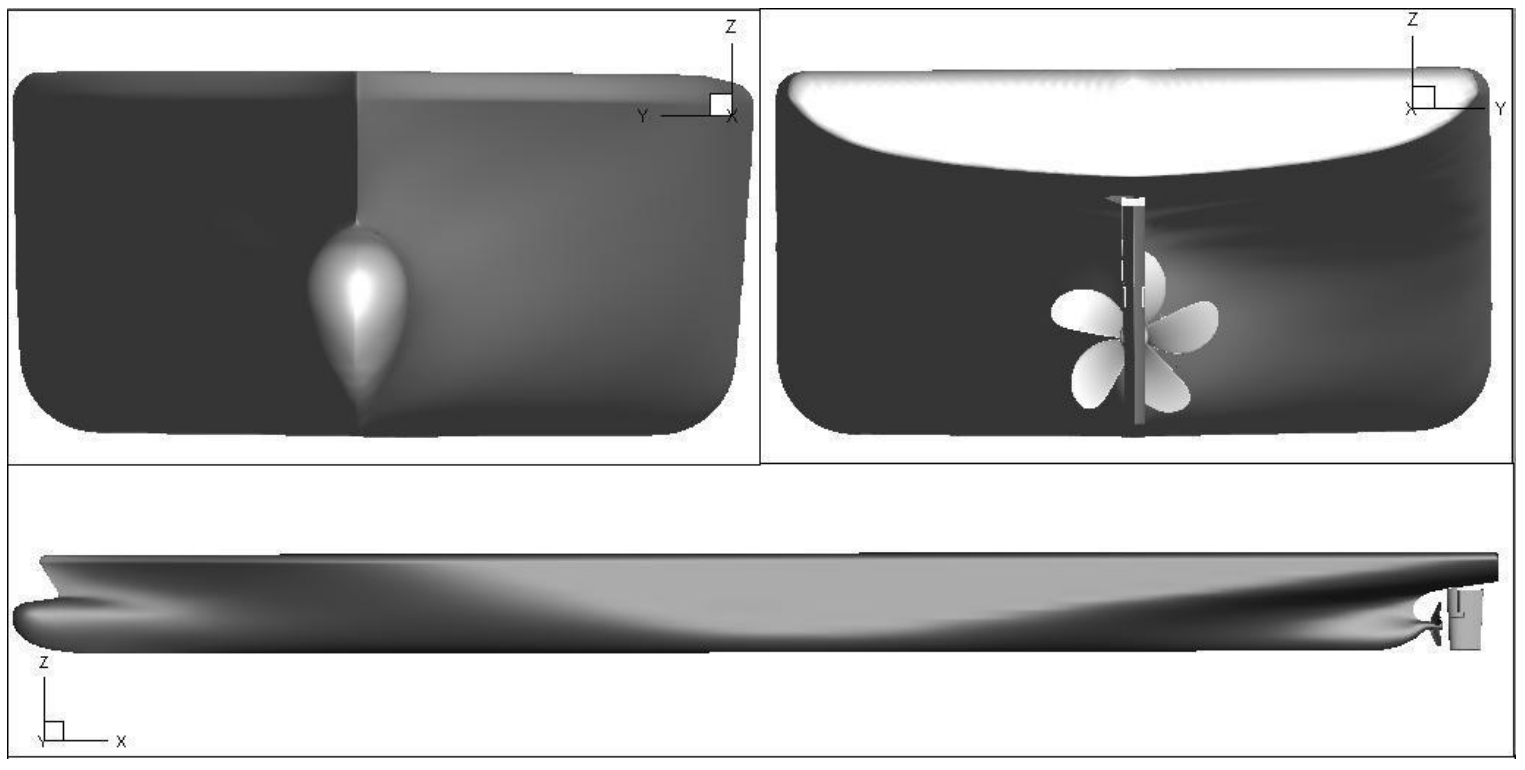

Figure 1: Fully appended KCS geometry. 


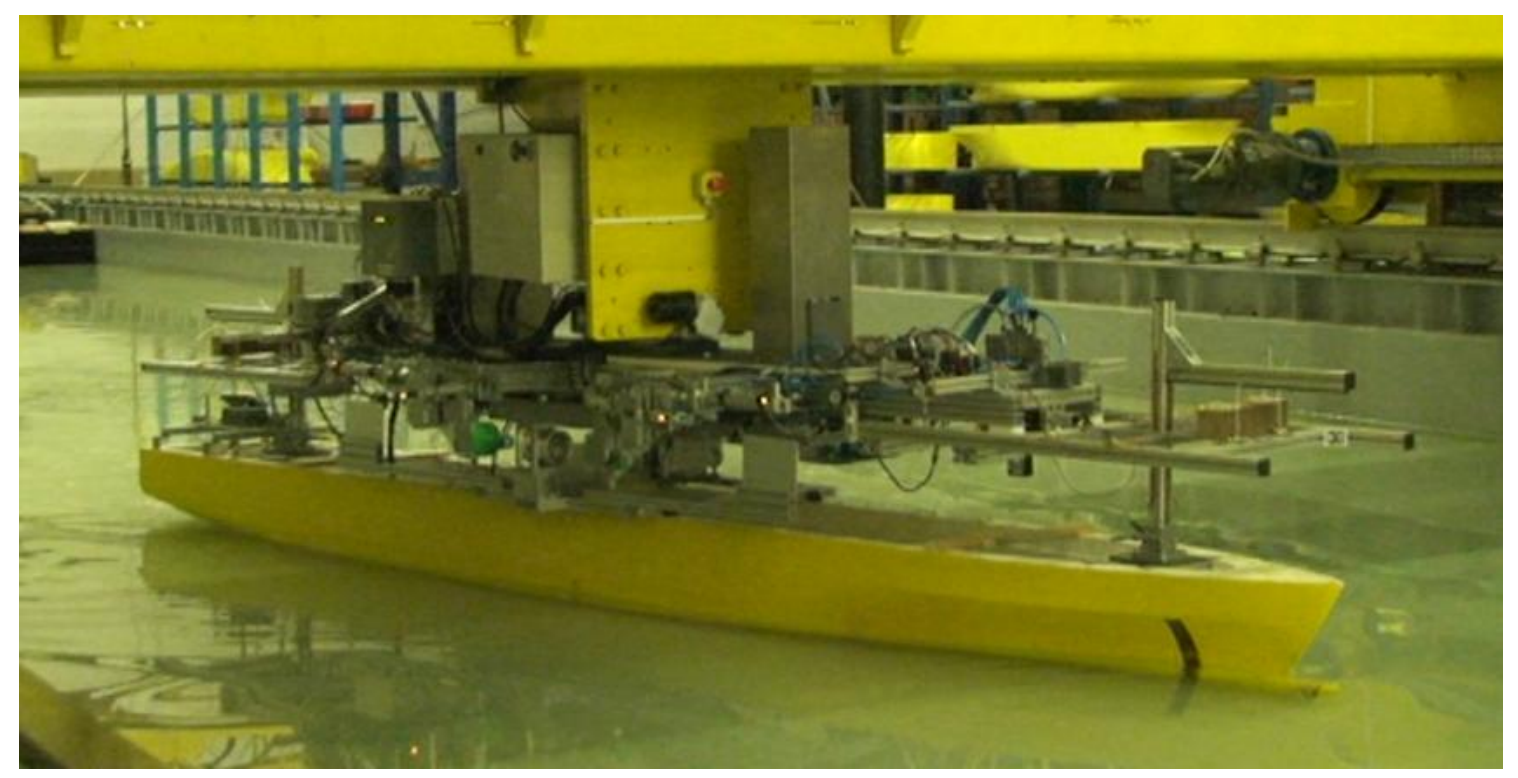

Figure 2: Ship model tested in free-running mode. 

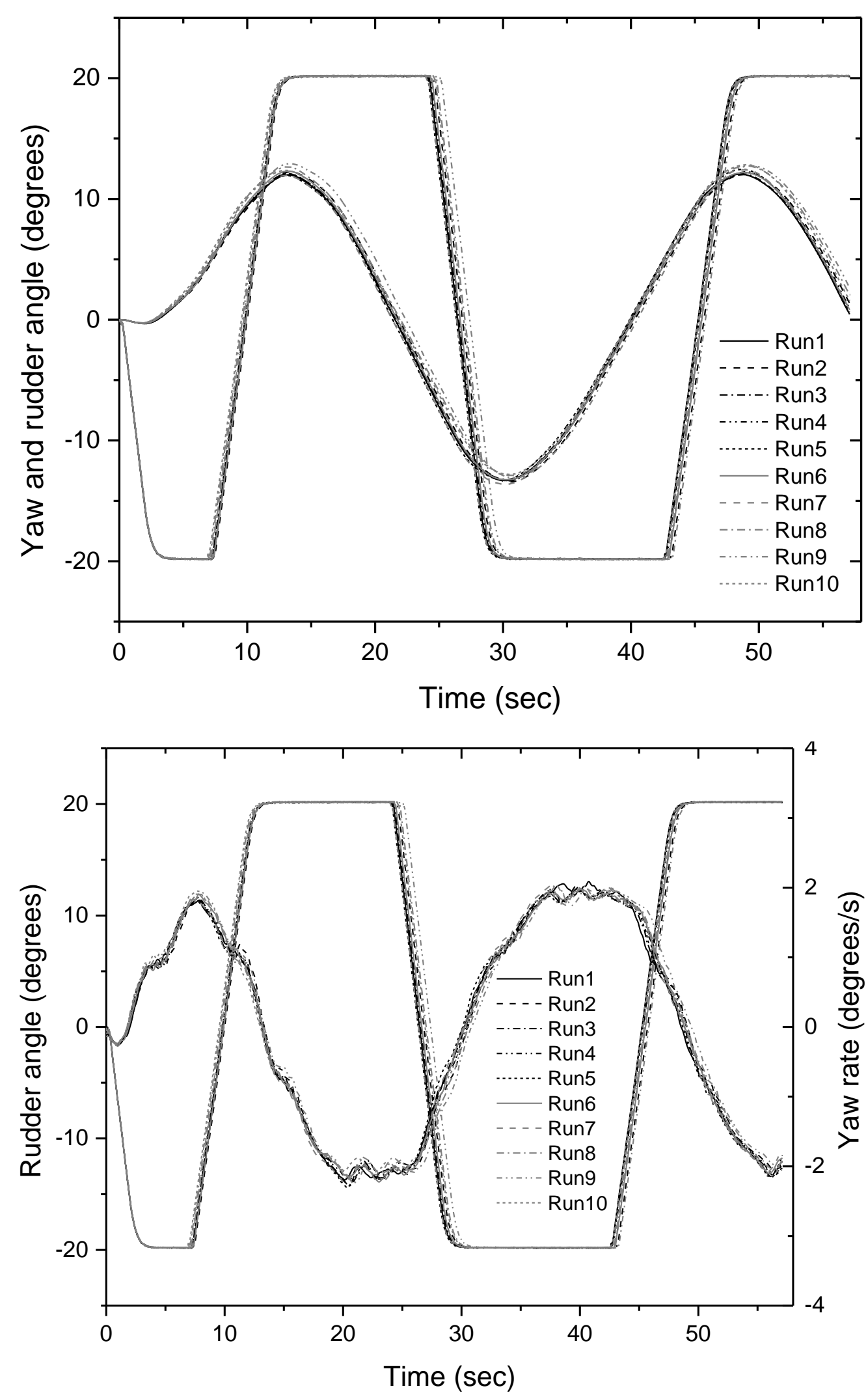
Figure 3: 20/5 zigzag maneuver with start to starboard: yaw and rudder angles and yaw rate time series measured during ten repeated tests.

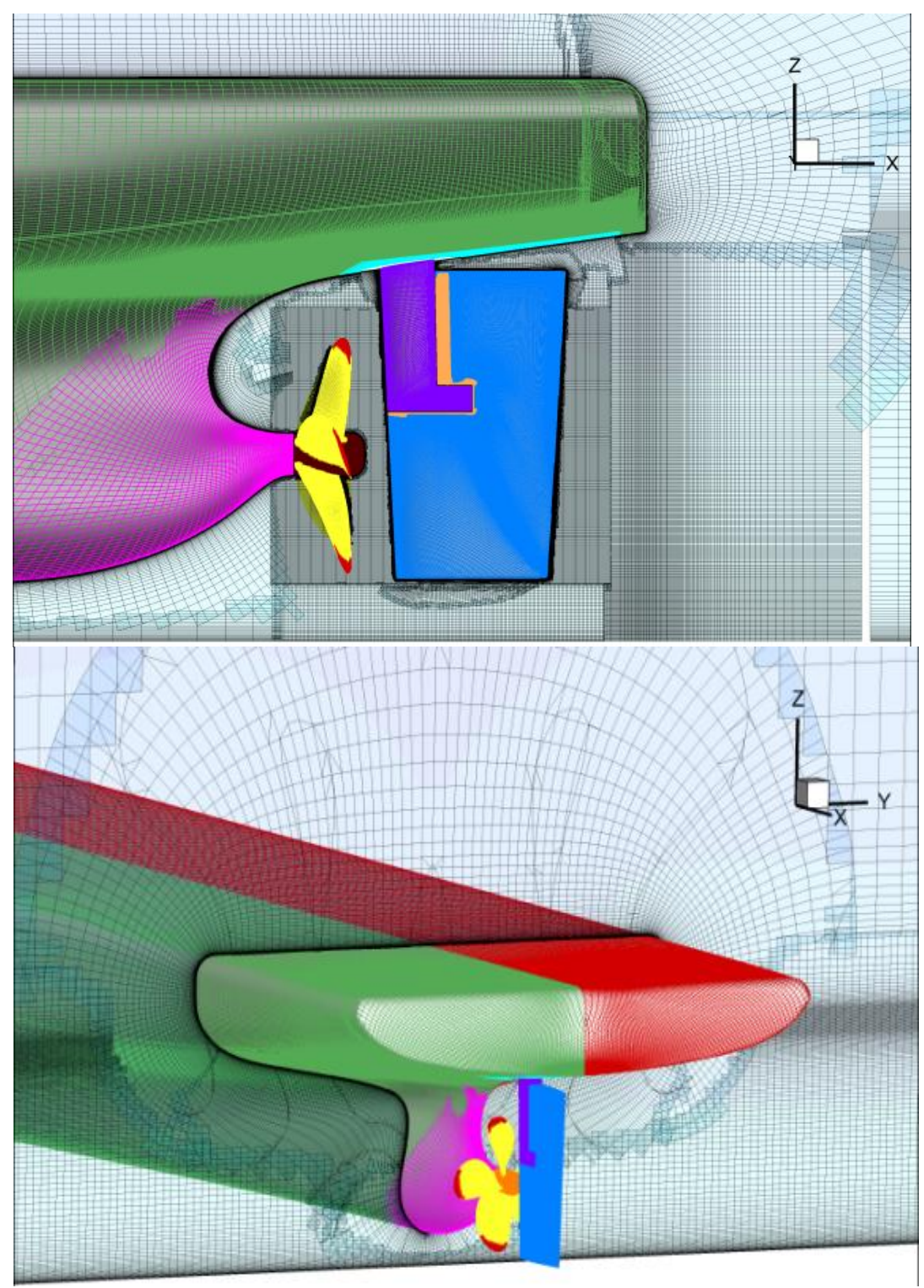

Fig. 4 Overset grid system topology, medium grid shown. 


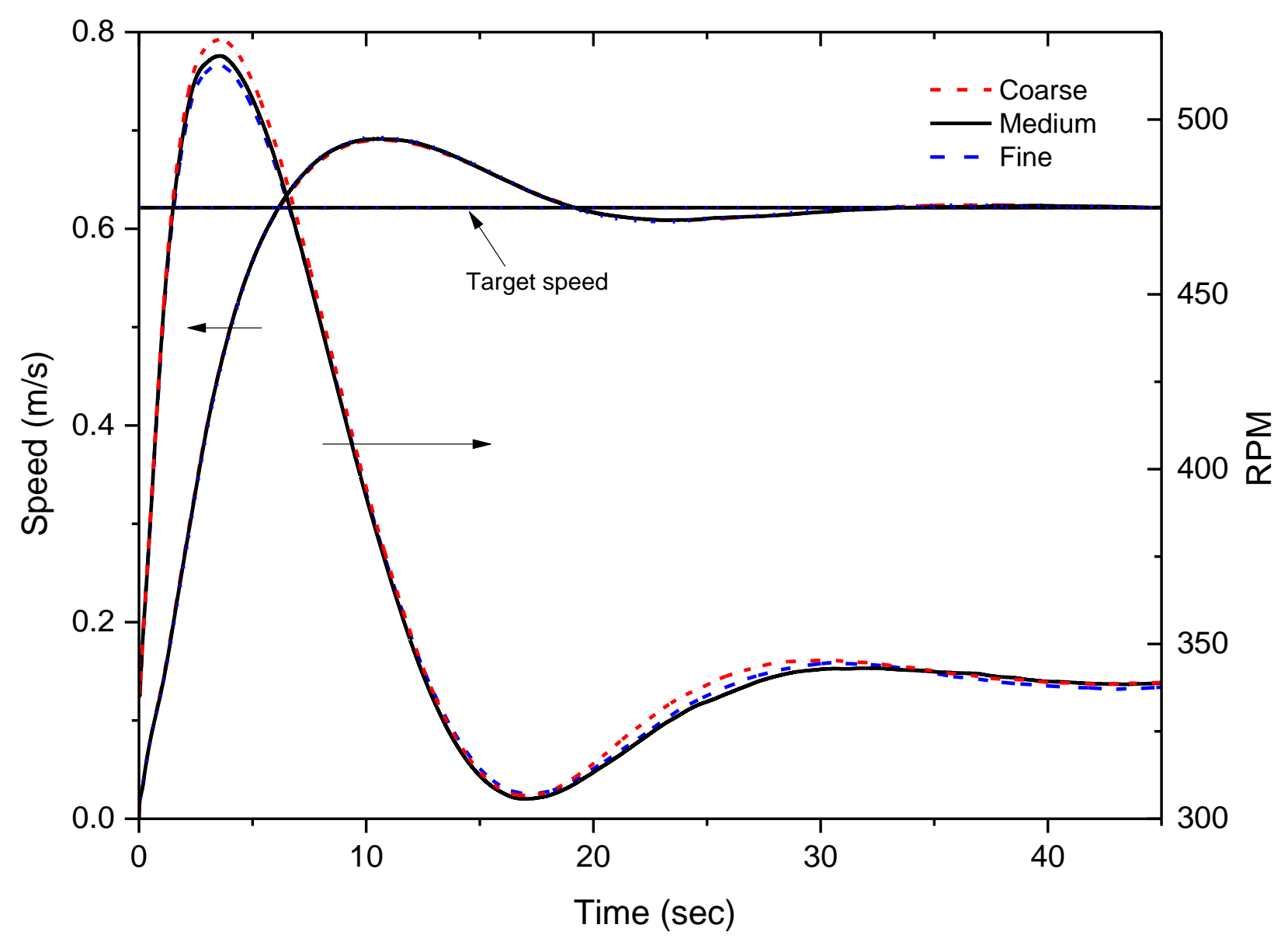

Fig. 5 Time history of ship speed and propeller rotational speed for the self-propulsion computation for Coarse, Medium and Fine grids. 


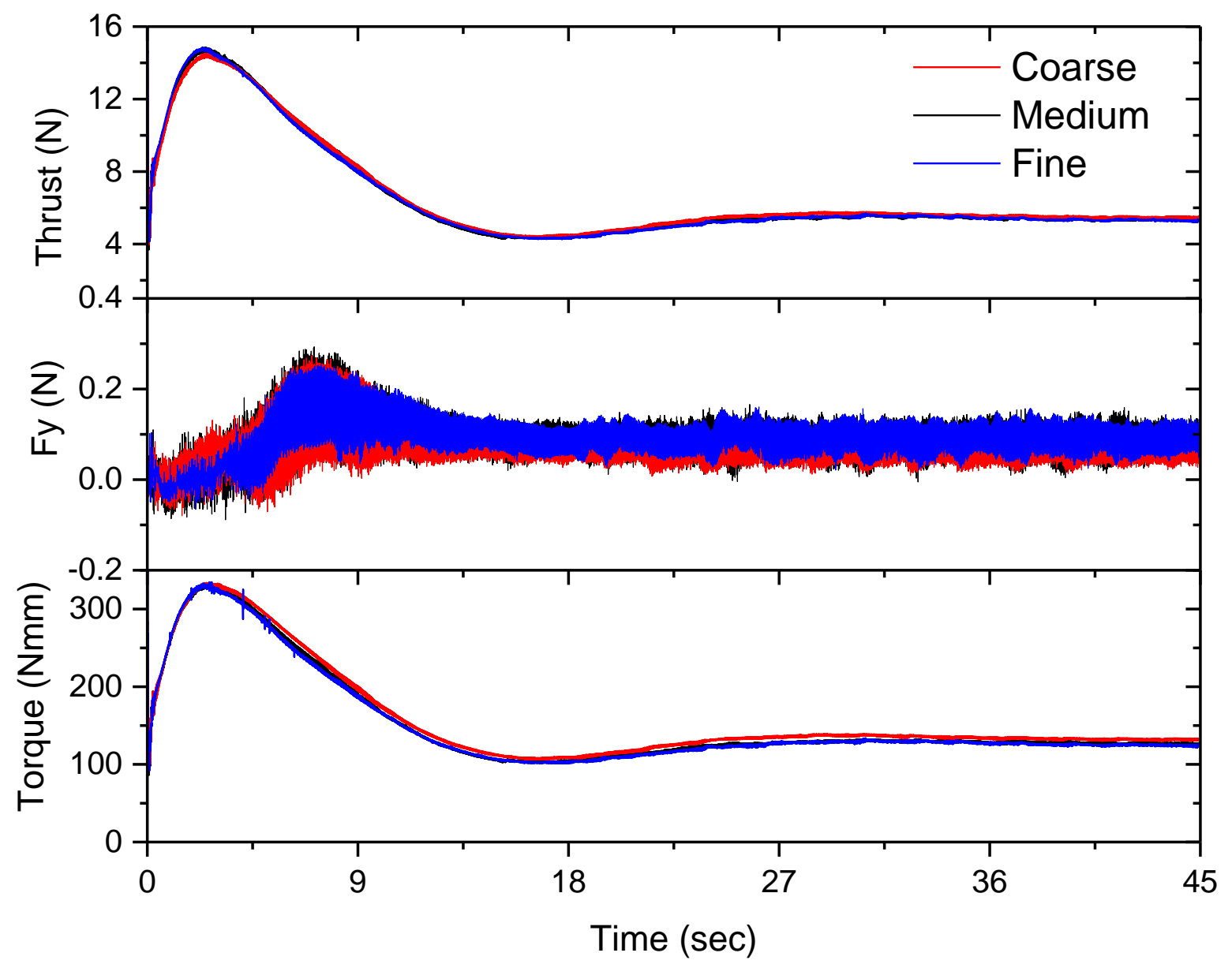

Fig. 6 Time history of propeller thrust, side force and torque for the self-propulsion computation for Coarse, Medium and Fine grids. 


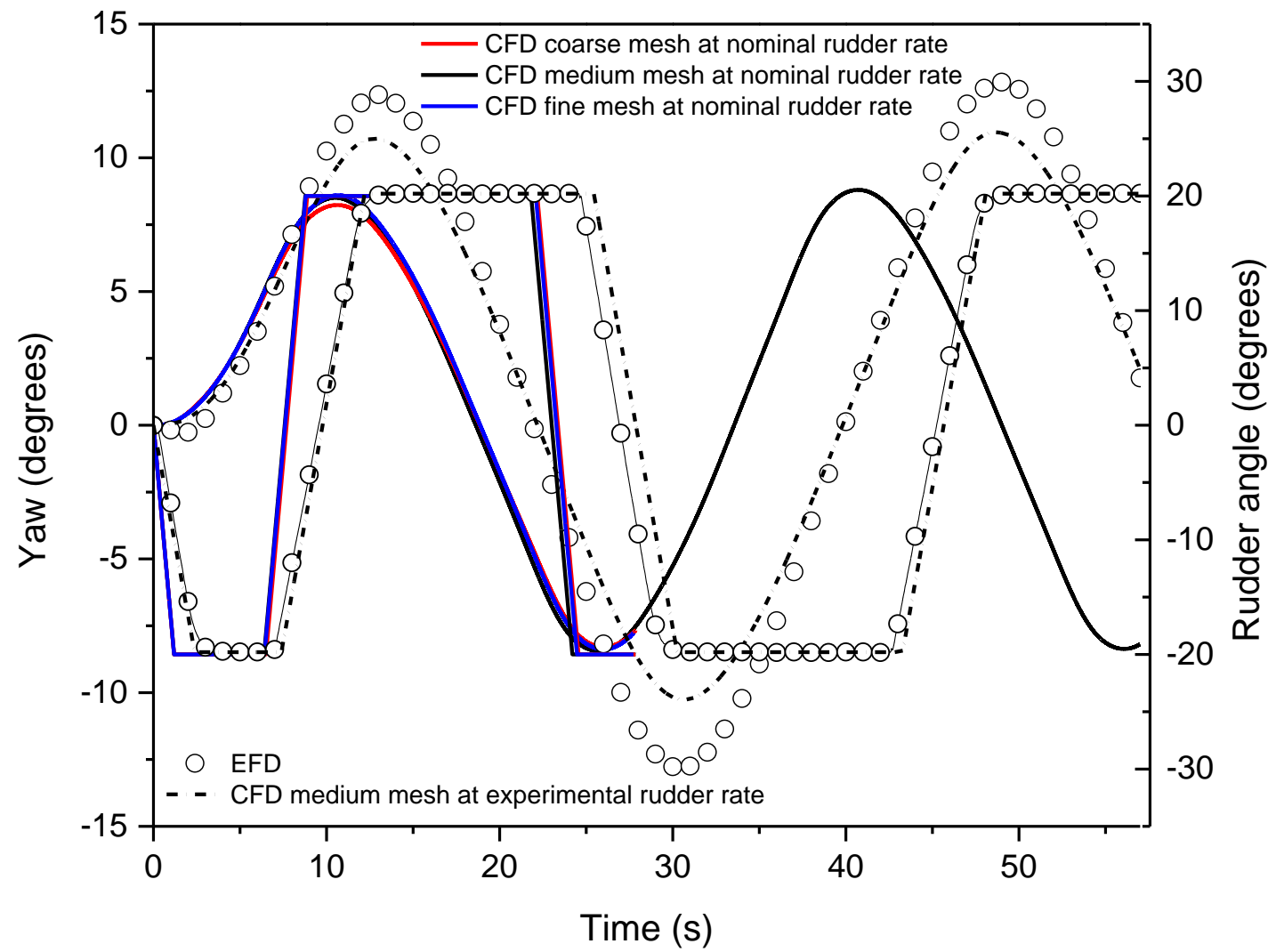

Fig. 7 Yaw and rudder angles for the 20/5 zigzag maneuver at nominal rudder rate for Coarse, Medium and Fine grids, and at the experimental rate for the medium grid and EFD. The experimental rudder rate is shown in lines with small white circle. 


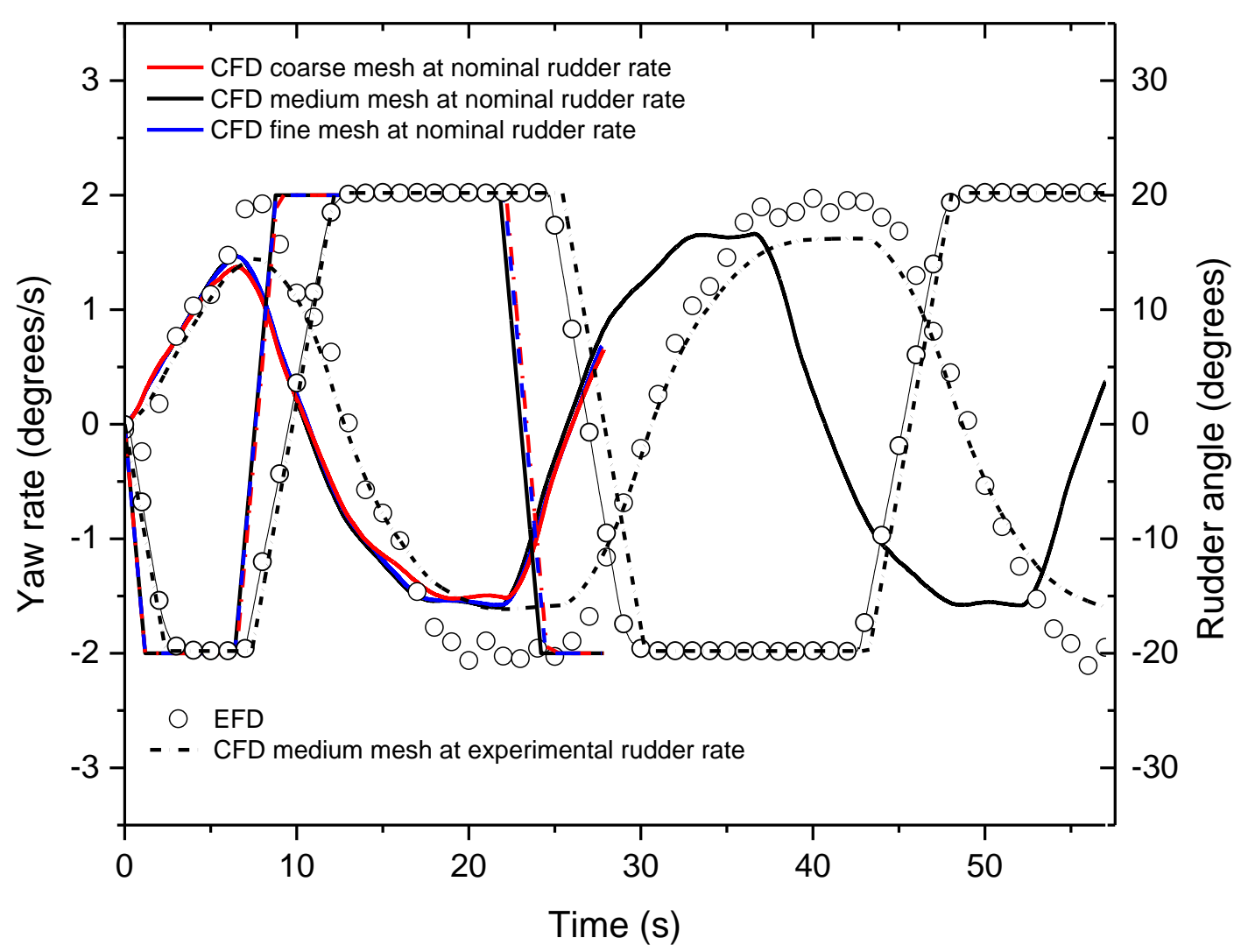

Fig. 8 Yaw rate and rudder angle for the 20/5 zigzag maneuver at nominal rudder rate for Coarse, Medium and Fine grids, and at the experimental rate for the medium grid and EFD. The experimental rudder rate is shown in lines with small white circle. 


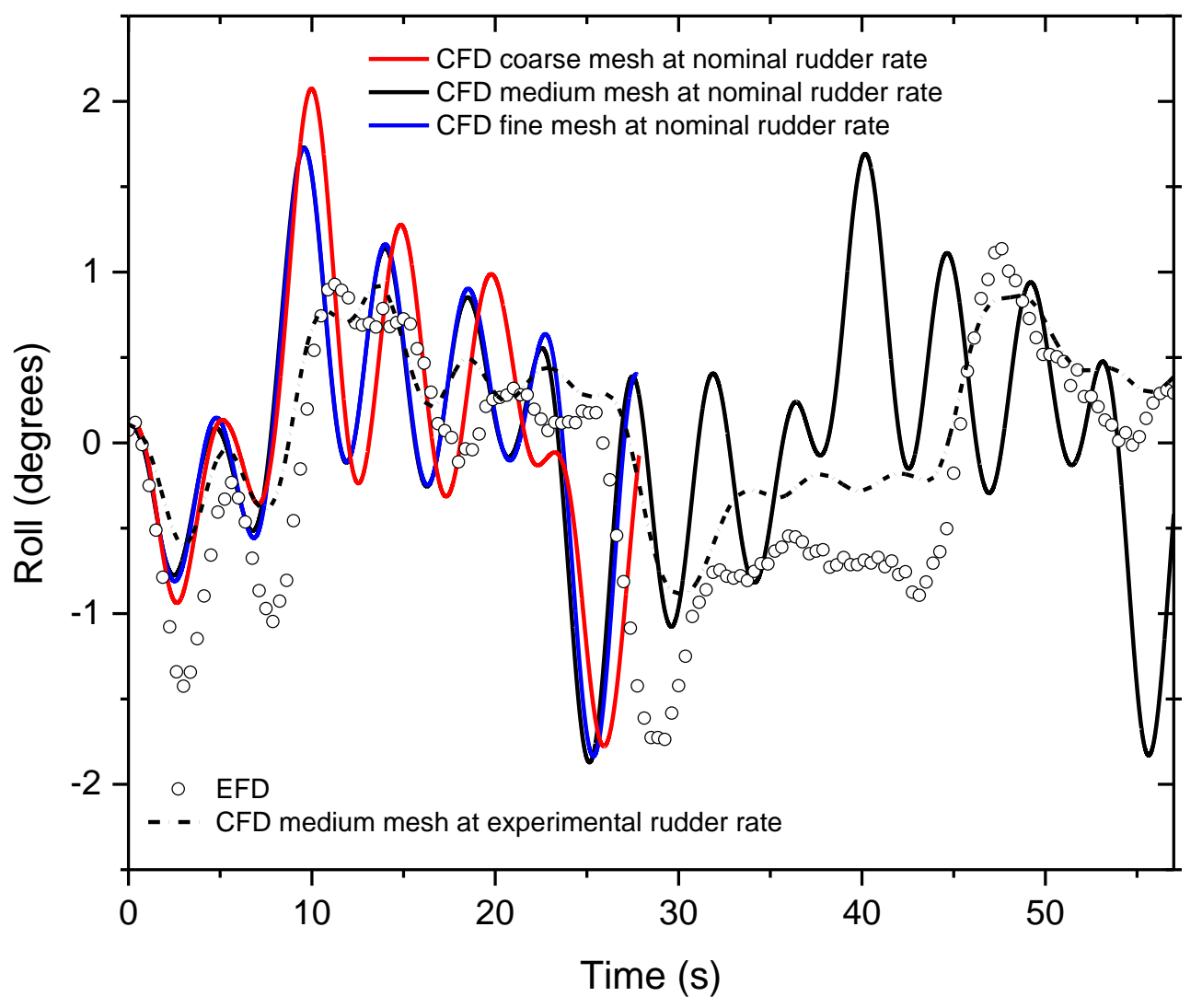

Fig. 9 Roll angle for the 20/5 zigzag maneuver at nominal rudder rate for Coarse, Medium and Fine grids, and at the experimental rate for the medium grid and EFD. 

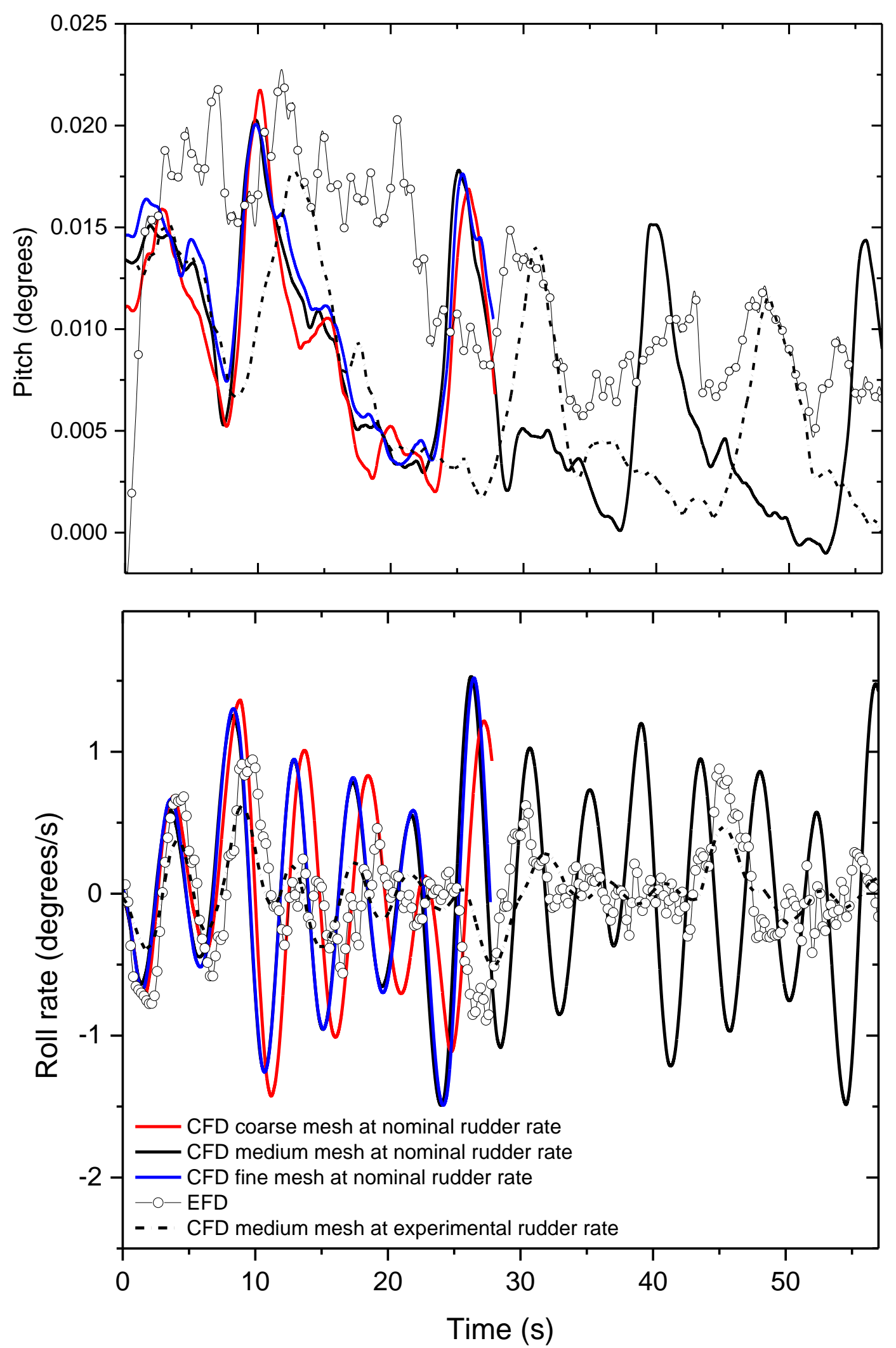
Fig. 10 Pitch angle and roll rate for the 20/5 zigzag maneuver at nominal rudder rate for Coarse, Medium and Fine grids, and at the experimental rate for the medium grid and EFD.

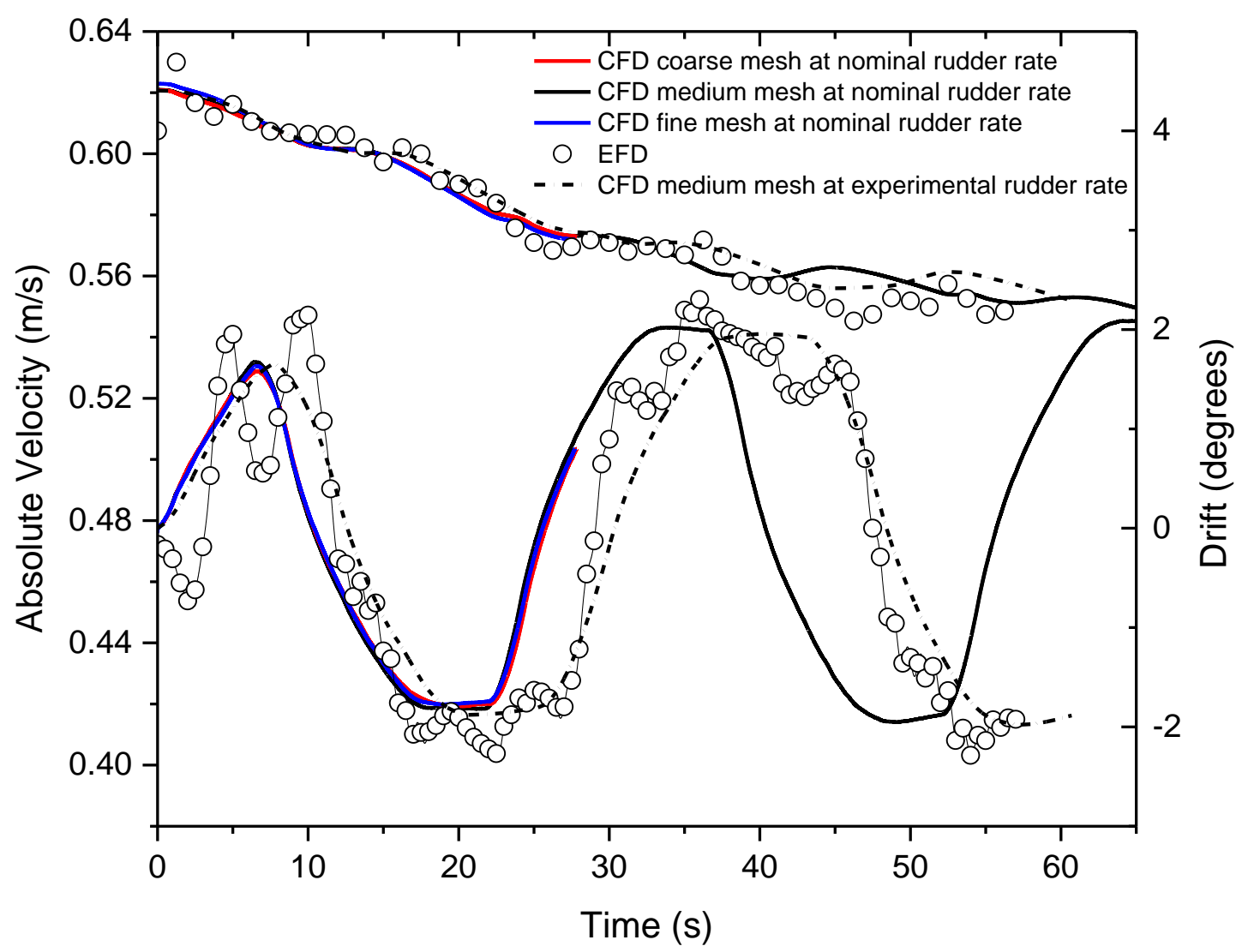

Fig. 11 Ship absolute velocity and drift angle for the 20/5 zigzag maneuver at nominal rudder rate for Coarse, Medium and Fine grids, and at the experimental rate for the medium grid and EFD. 


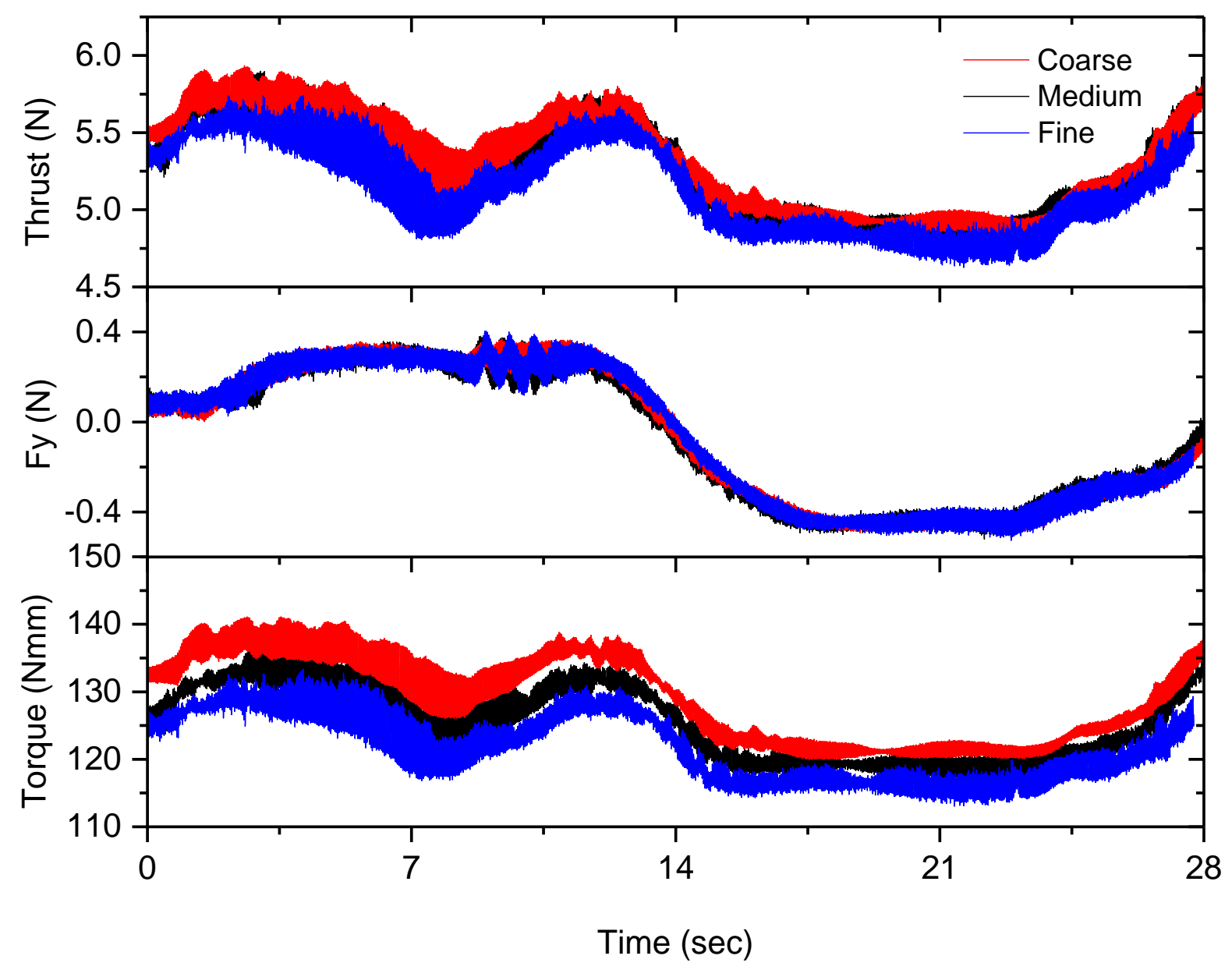

Fig. 12 Time histories of propeller thrust, side force and torque for the 20/5 zigzag maneuver at nominal rudder rate for Coarse, Medium and Fine grids. 


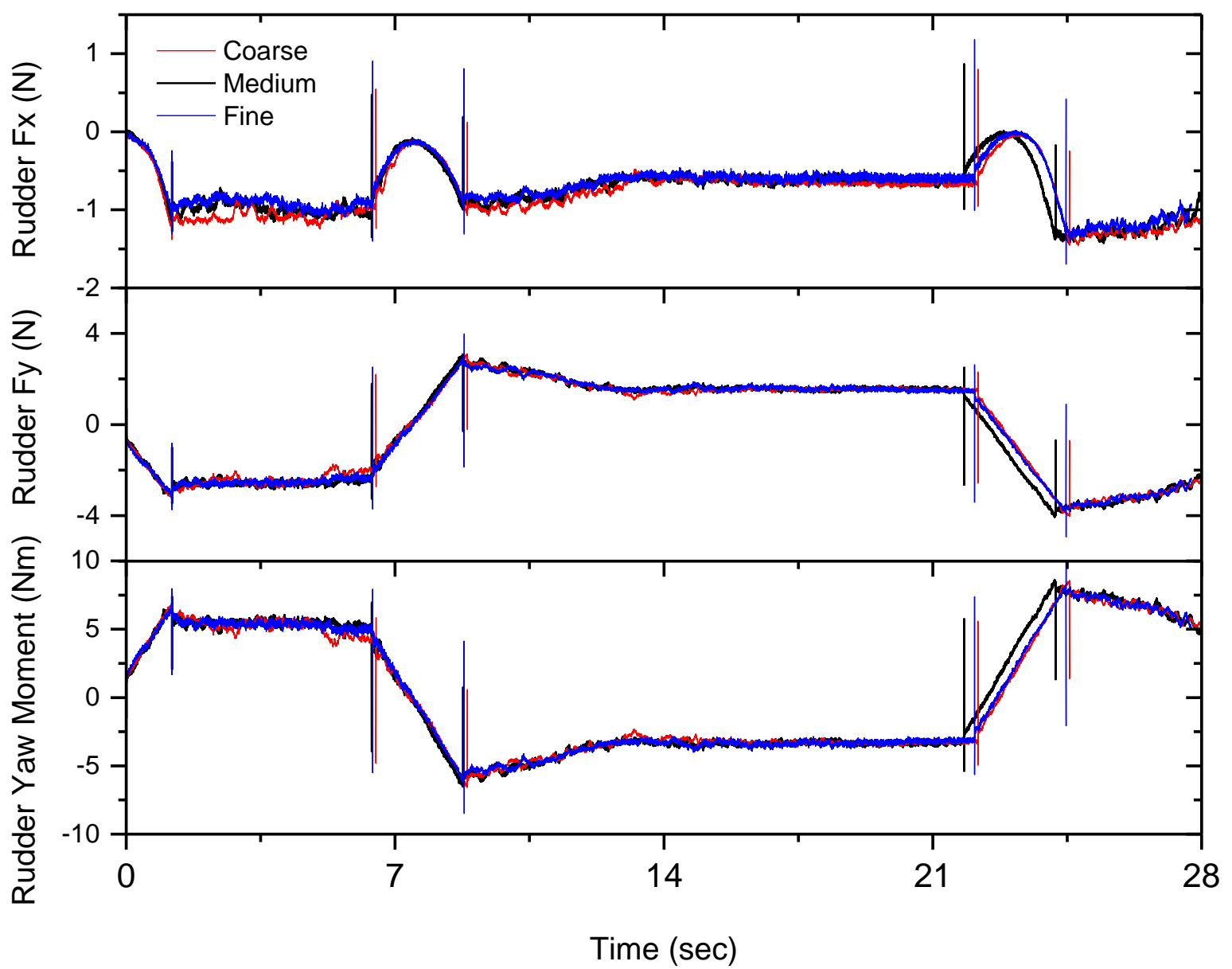

Fig. 13 Time histories of rudder X-force, Y-force and yaw moment for the 20/5 zigzag maneuver at nominal rudder rate for Coarse, Medium and Fine grids. 

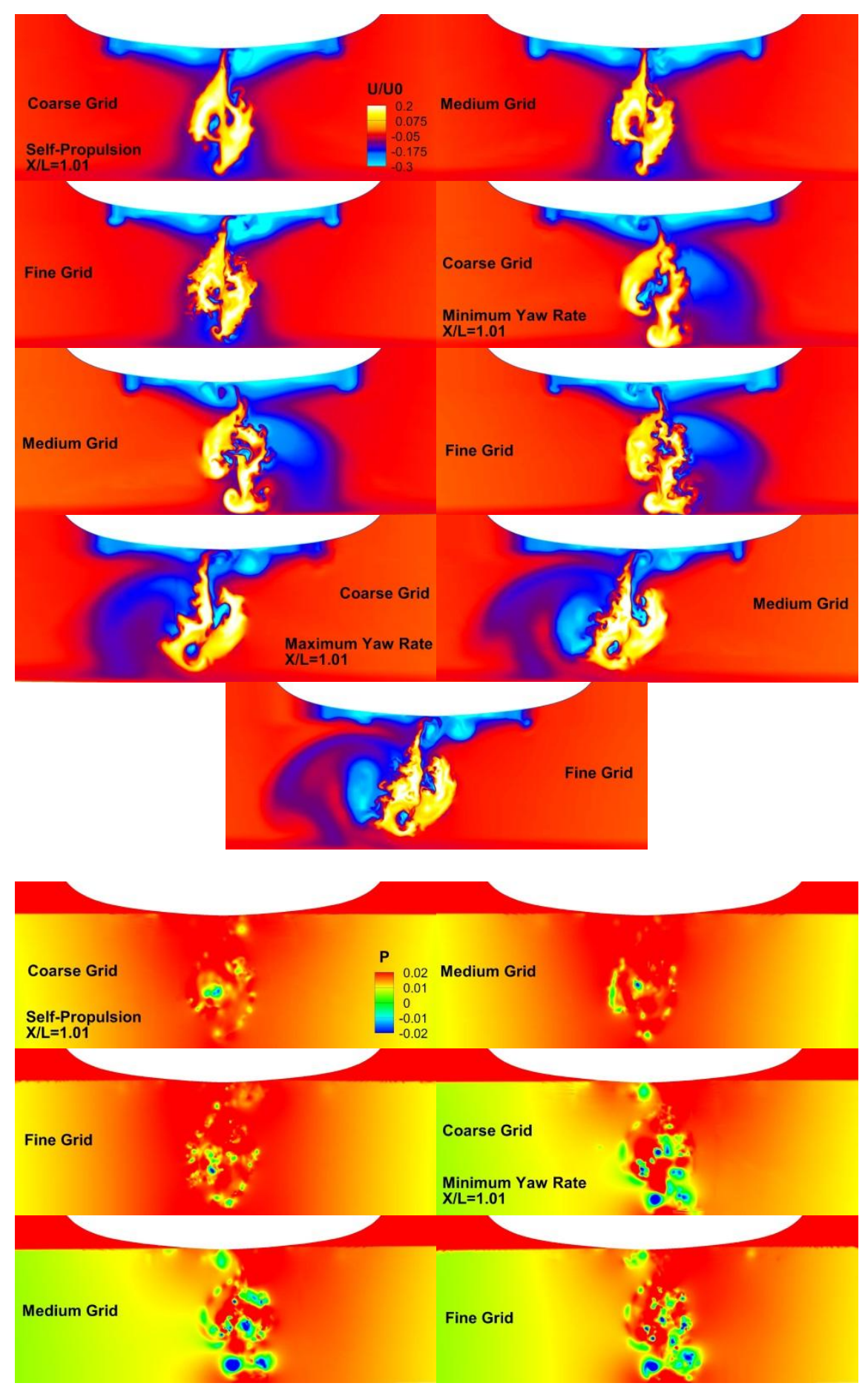


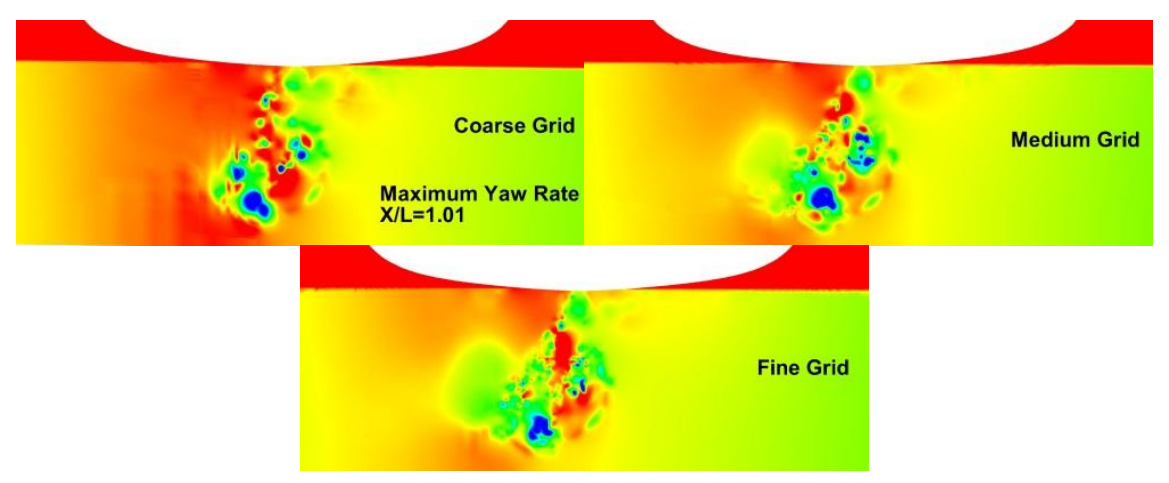

Fig. 14 Velocity (top figures) and Pressure (bottom figures) contours at $\mathrm{X} / \mathrm{L}=1.01$ at self-propulsion (top), minimum yaw rate (center) and maximum yaw rate (bottom) for coarse, medium and fine grids. Vertical axis range: $-0.05<\frac{Z}{L}<0.1$, horizontal axis range: $-0.09<Y / L<0.09$.

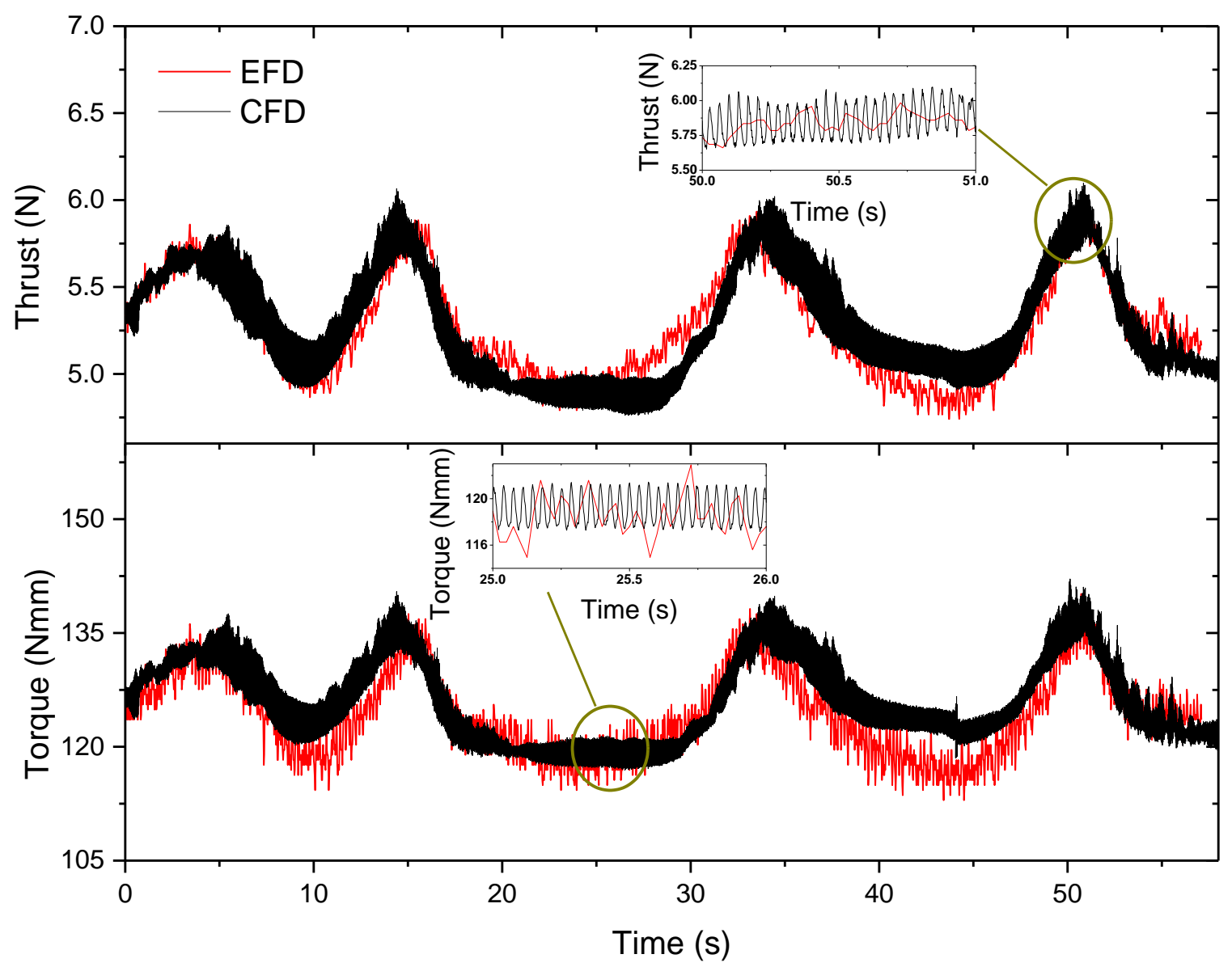

Figure 15: Propeller thrust and torque for the 20/5 zigzag maneuvers at the experimental rudder rate for medium grid and EFD. 


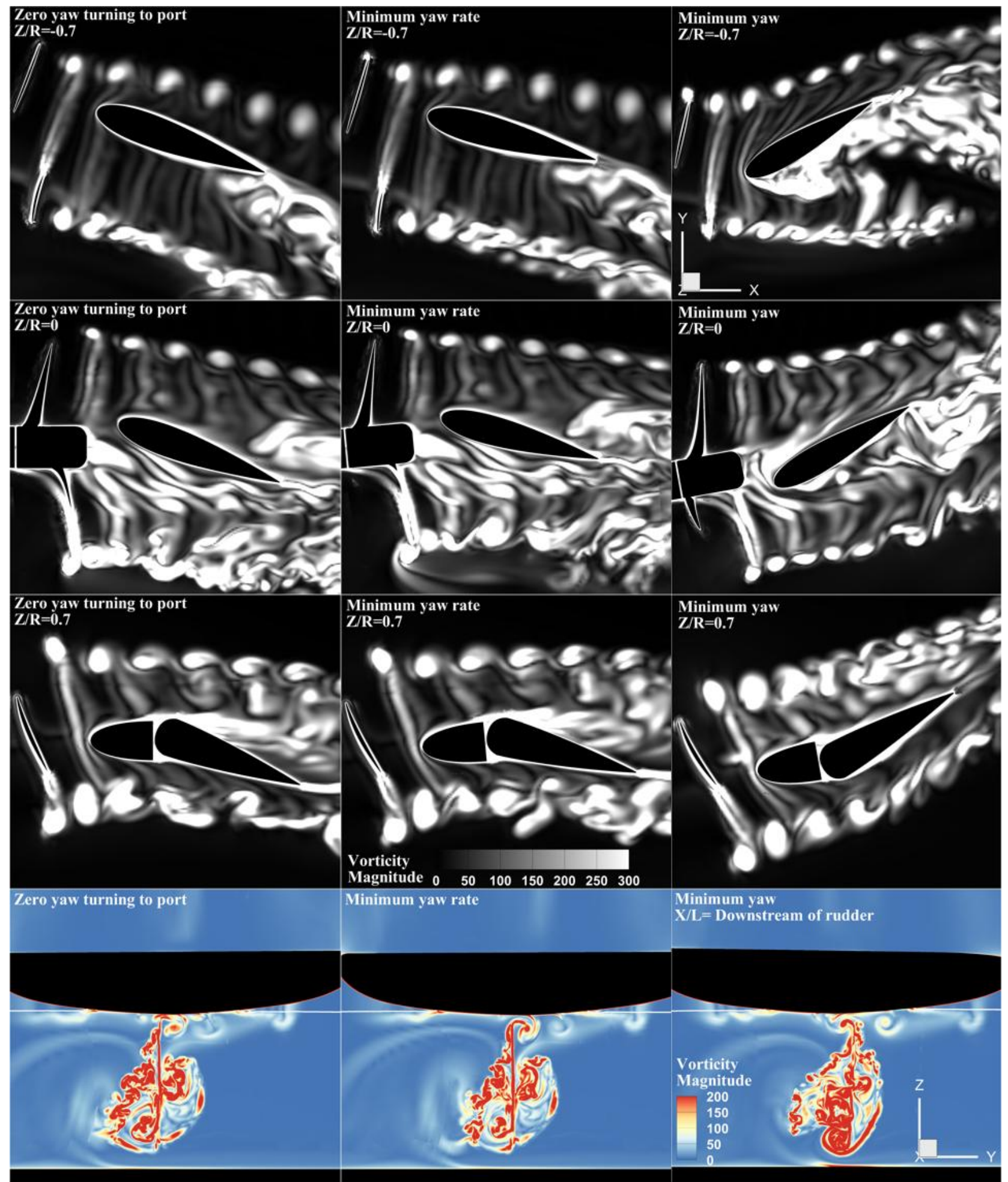

Figure 16: Horizontal sections $0.7 R$ below the propeller axis $(Z / R=-0.7$, top row), at the propeller axis $(Z / R=0$, second row), and $0.7 R$ above the propeller axis $(Z / R=0.7$, third row $)$, and axial cross section at the trailing edge of the rudder (bottom row), showing vorticity magnitude at instantaneous points of zero yaw turning to port, minimum yaw rate and minimum yaw. 


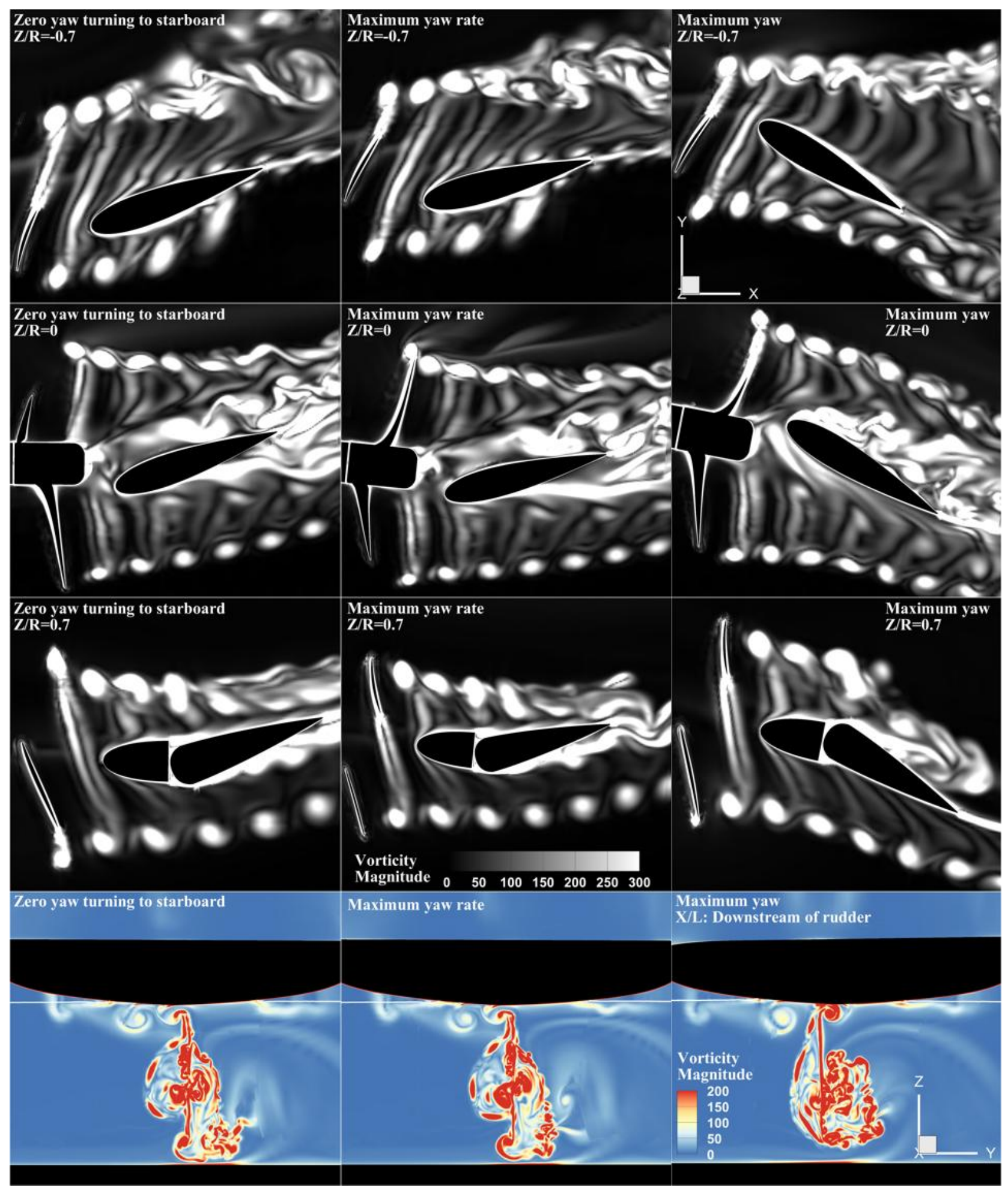

Figure 17: Horizontal sections $0.7 R$ below the propeller axis $(Z / R=-0.7$, top row), at the propeller axis $(Z / R=0$, second row), and $0.7 R$ above the propeller axis $(Z / R=0.7$, third row), and axial cross section at the trailing edge of the rudder (bottom row), showing vorticity magnitude at instantaneous points of zero yaw turning to starboard, maximum yaw rate and maximum yaw. 


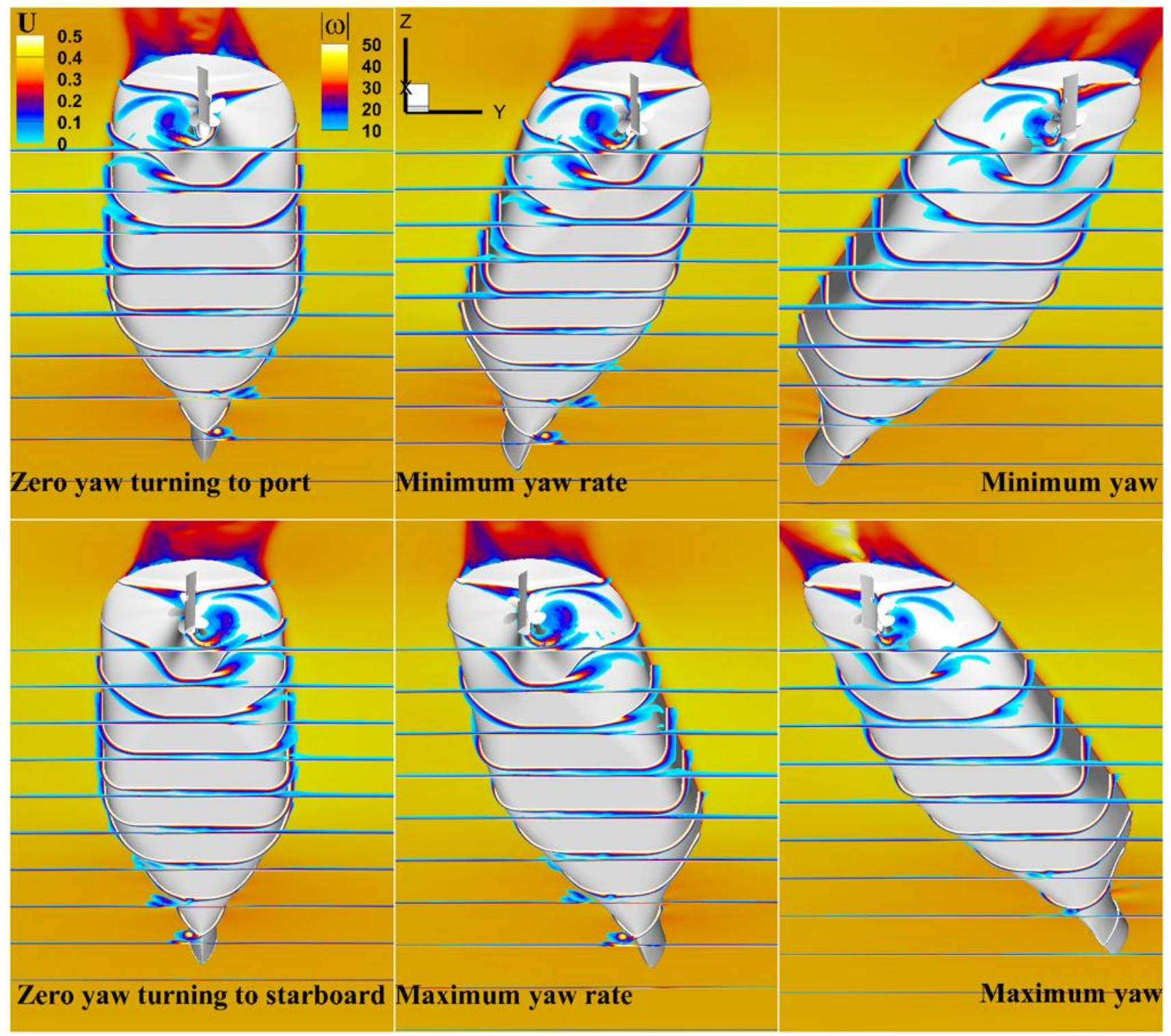

Figure 18: Cross sections colored with vorticity magnitude showing the boundary layer at instantaneous points of zero yaw turning to port, minimum yaw rate, minimum yaw, zero yaw turning to starboard, maximum yaw rate and maximum yaw. Free surface colored with velocity. 


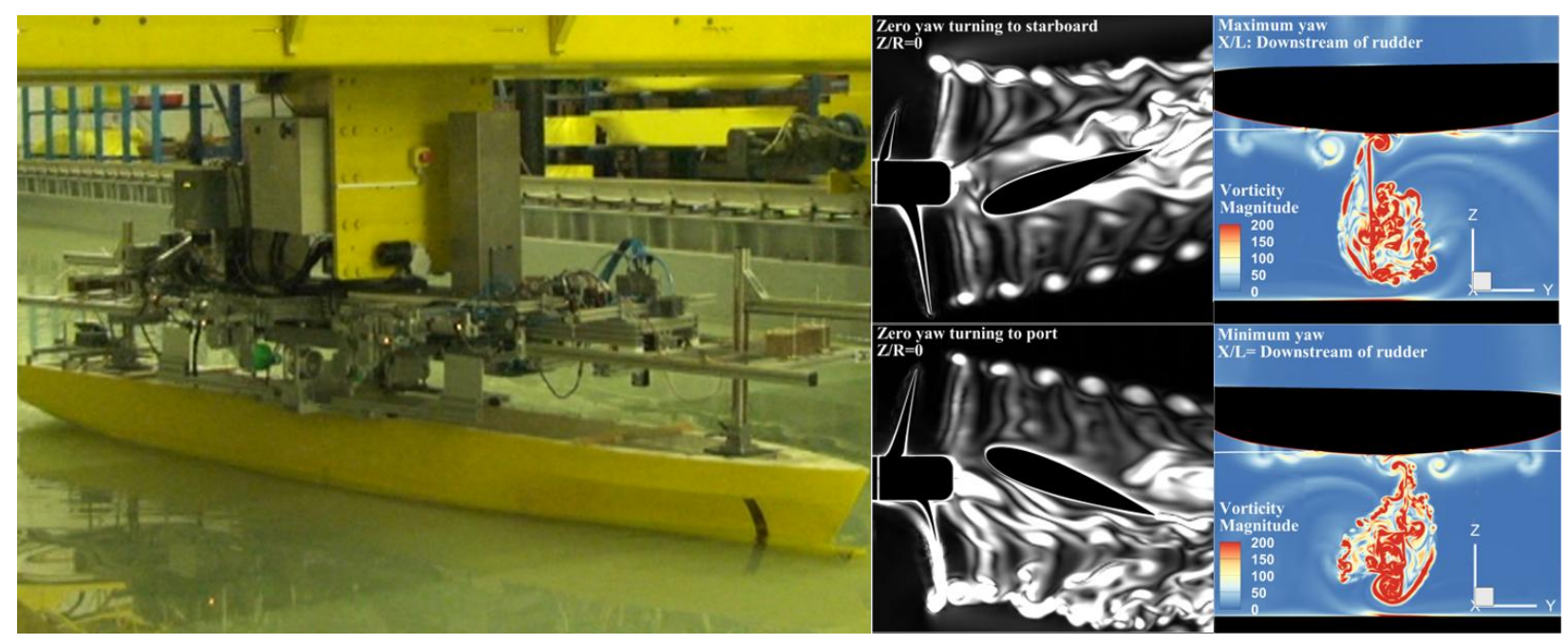

Graphical Abstract 